MATHEMATICS OF COMPUTATION

Volume 73, Number 247, Pages 1041-1066

S 0025-5718(03)01578-3

Article electronically published on August 19, 2003

\title{
CONVERGENCE OF NONCONFORMING $V$-CYCLE AND F-CYCLE MULTIGRID ALGORITHMS FOR SECOND ORDER ELLIPTIC BOUNDARY VALUE PROBLEMS
}

\author{
SUSANNE C. BRENNER
}

\begin{abstract}
The convergence of $V$-cycle and $F$-cycle multigrid algorithms with a sufficiently large number of smoothing steps is established for nonconforming finite element methods for second order elliptic boundary value problems.
\end{abstract}

\section{INTRODUCTION}

Let $\Omega \subset \mathbb{R}^{2}$ be a bounded polygonal domain. Consider the variational problem of finding $u \in H_{0}^{1}(\Omega)$ such that

$$
a(u, v)=F(v) \quad \forall v \in H_{0}^{1}(\Omega),
$$

where $F \in H^{-1}(\Omega)$ and

$$
a(v, w)=\int_{\Omega}\left[\sum_{i, j=1}^{2} a_{i j}(x) \frac{\partial v}{\partial x_{i}} \frac{\partial w}{\partial x_{j}}+r(x) v w\right] d x .
$$

We assume that $a_{i j}, r \in C^{1}(\bar{\Omega}), a_{12}=a_{21}, r \geq 0$ on $\bar{\Omega}$, and

$$
\sum_{i, j=1}^{2} a_{i j}(x) \xi_{i} \xi_{j} \geq c\left(\xi_{1}^{2}+\xi_{2}^{2}\right) \quad \forall x \in \bar{\Omega}, \xi_{1}, \xi_{2} \in \mathbb{R},
$$

where $c$ is a positive constant. Under these conditions the bilinear form $a(\cdot, \cdot)$ on $H_{0}^{1}(\Omega) \times H_{0}^{1}(\Omega)$ is bounded and coercive, and (1.1) has a unique solution.

It is well known (cf. $\S 5$.C and $\S 14$.A of [32]) that there exists $\alpha \in\left(\frac{1}{2}, 1\right]$ such that the solution $u$ of (1.1) belongs to $H^{1+\alpha}(\Omega) \cap H_{0}^{1}(\Omega)$ whenever $F \in H^{-1+\alpha}(\Omega)$ and

$$
\|u\|_{H^{1+\alpha}(\Omega)} \leq C_{\Omega}\|F\|_{H^{-1+\alpha}(\Omega)} .
$$

Approximate solutions of the variational problem (1.1) can be obtained by the finite element method (cf. 30, 25]). The resulting symmetric positive definite systems are sparse and can be solved efficiently by multigrid algorithms (cf. 36, [39], 7], 16], 49]). For the symmetric $V$-cycle algorithm with equal numbers of presmoothing and post-smoothing steps, the classical result by Braess and Hackbusch

Received by the editor May 29, 2001 and, in revised form, January 10, 2003.

2000 Mathematics Subject Classification. Primary 65N55, 65 N30.

Key words and phrases. Multigrid, $V$-cycle, $F$-cycle, nonconforming finite elements.

This work was supported in part by the National Science Foundation under Grant No. DMS00-74246.

(C)2003 American Mathematical Society 
(cf. [35, [5], 3], 39, [9]) states that, in the case where $\alpha=1$ (i.e., when $\Omega$ is convex),

$$
\gamma_{k} \leq \frac{C}{C+m} \quad \text { for } m=1,2, \ldots,
$$

where $\gamma_{k}$ is the contraction number of the $k$-th level $V$-cycle algorithm in the norm

$$
\|\cdot\|_{a}=\sqrt{a(\cdot, \cdot)},
$$

$m$ is the number of pre-smoothing and post-smoothing steps, and $C$ (with or without subscripts) henceforth denotes a generic positive constant which is independent of $k$ and $m$.

The case where $\frac{1}{2}<\alpha<1$ (i.e., when $\Omega$ has re-entrant corners) is more subtle. It was not until the early nineties, after a multiplicative theory for multilevel methods (cf. [13, 12]) had been developed, that the following result was established (cf. [57, 53, 10, 56, 11, 34, 41]):

$$
\gamma_{k} \leq \delta,
$$

where $\delta \in(0,1)$ is independent of $k$. The asymptotic behavior of $\gamma_{k}$ with respect to $m$ was studied in 22 by an additive convergence theory. It was shown that, for the $\mathrm{P}_{1}$ finite element and with the Richardson relaxation scheme as smoother,

$$
\gamma_{k} \leq \frac{C}{m^{\alpha}} \quad \text { for } m \geq m_{0},
$$

where the positive integer $m_{0}$ is independent of $k$. It then follows easily from (1.6) and (1.7) that

$$
\gamma_{k} \leq \frac{C}{C+m^{\alpha}} \quad \text { for } m=1,2, \ldots
$$

In other words, a complete generalization of the result of Braess and Hackbusch to the case of less than full elliptic regularity has been obtained. The results in [22] were generalized to include other smoothers in [23].

In this paper we extend the theory in 22 to nonconforming finite elements and establish the same estimate (1.7). Since the $V$-cycle algorithm for nonconforming finite elements in general does not converge uniformly for $m=1$, the estimate (1.7) is the best possible general estimate for such methods. As a by-product we also obtain similar estimates for nonconforming $F$-cycle algorithms (cf. [17, 40, [51, [49]). As far as we know, this is the first convergence result for nonconforming $F$-cycle algorithms, even though it has been known for some time (cf. [50]) that these algorithms perform very well in practice. The theory developed in this paper can also be applied to fourth order problems (cf. [58).

We also note in passing that most of the general convergence results for nonconforming multigrid algorithms were obtained for the $W$-cycle algorithm and the variable $V$-cycle preconditioner (cf. [18, [14, [21] and the references therein, and also [4], 47] for the cascadic multigrid algorithm). The only existent nonconforming $V$-cycle convergence results involve either special elements (cf. [54, [45], [46]), conforming finite element spaces on coarse grids (cf. [52], [55]), or the suboptimal Galerkin approach (cf. [29]).

The rest of the paper is organized as follows. In Section 2 we set up $V$-cycle and $F$-cycle algorithms for nonconforming finite elements in an abstract setting. The assumptions for the convergence theory are stated in Section 3 We then prove a strengthened Cauchy-Schwarz inequality in Section 4 The estimate (1.7) for $V$-cycle and $F$-cycle algorithms is established in Section [5. In Section 6 we show 
that the assumptions in Section 3 can be verified within an abstract framework for nonconforming multigrid methods. Applications to concrete nonconforming finite elements are then given in Section 7 .

For future reference we state here two simple inequalities:

$$
\begin{aligned}
2 a b & \leq(\theta a)^{2}+\left(\theta^{-1} b\right)^{2} & & \forall a, b \in \mathbb{R}, \theta \in(0,1), \\
(a+b)^{2} & \leq\left(1+\theta^{2}\right) a^{2}+\left(1+\theta^{-2}\right) b^{2} & & \forall a, b \in \mathbb{R}, \theta \in(0,1) .
\end{aligned}
$$

\section{NonCONFORMing $V$-CYCLE AND $F$-CYCle MUltigrid ALGORIthmS}

Let $\mathcal{T}_{1}$ be a triangulation of $\Omega$ and let the triangulations $\mathcal{T}_{k}$, for $k=2,3, \ldots$, be obtained by successive regular subdivisions. The mesh size $h_{k}$ of $\mathcal{T}_{k}$ therefore satisfies the relation

$$
h_{k}=2 h_{k+1} \quad \text { for } k=1,2, \ldots .
$$

The discontinuous energy space $H^{1}\left(\mathcal{T}_{k}\right)$ associated with $\mathcal{T}_{k}$ is defined by

$$
H^{1}\left(\mathcal{T}_{k}\right)=\left\{v \in L_{2}(\Omega):\left.v\right|_{T} \in H^{1}(T) \quad \forall T \in \mathcal{T}_{k}\right\}
$$

We define the nonconforming variational form $a_{k}(\cdot, \cdot)$ on $H^{1}\left(\mathcal{T}_{k}\right)$ by

$$
a_{k}(v, w)=\sum_{T \in \mathcal{T}_{k}} \int_{T}\left[\sum_{i . j=1}^{2} a_{i j}(x) \frac{\partial v}{\partial x_{i}} \frac{\partial w}{\partial x_{j}}+r(x) v w\right] d x \quad \forall v, w \in H^{1}\left(\mathcal{T}_{k}\right)
$$

and the corresponding nonconforming energy (semi-)norm $\|\cdot\|_{a_{k}}$ by

$$
\|v\|_{a_{k}}=\sqrt{a_{k}(v, v)} \quad \forall v \in H^{1}\left(\mathcal{T}_{k}\right) .
$$

Note that $H^{1}\left(\mathcal{T}_{1}\right) \subset H^{1}\left(\mathcal{T}_{2}\right) \subset \cdots$ and

$$
\|v\|_{a_{k-1}}=\|v\|_{a_{k}} \quad \forall v \in H^{1}\left(\mathcal{T}_{k-1}\right) .
$$

Moreover, it follows from the boundedness and coercivity of $a(\cdot, \cdot)$ and the Poincaré inequality that

$$
\|\zeta\|_{a_{k}}=\|\zeta\|_{a} \approx\|\zeta\|_{H^{1}(\Omega)} \approx|\zeta|_{H^{1}(\Omega)} \quad \forall \zeta \in H_{0}^{1}(\Omega) .
$$

Let $V_{k} \subset H^{1}\left(\mathcal{T}_{k}\right)$ be a nonconforming finite element space associated with $\mathcal{T}_{k}$ such that $a_{k}(\cdot, \cdot)$ is positive definite on $V_{k}$, and let $(\cdot, \cdot)_{k}$ be a discrete inner product on $V_{k}$. We can then represent $a_{k}(\cdot, \cdot)$ by the operator $A_{k}: V_{k} \longrightarrow V_{k}$ defined by

$$
\left(A_{k} v_{1}, v_{2}\right)_{k}=a_{k}\left(v_{1}, v_{2}\right) \quad \forall v_{1}, v_{2} \in V_{k} .
$$

Note that $A_{k}$ is symmetric positive definite with respect to $(\cdot, \cdot)_{k}$ and the following relation holds:

$$
a_{k}\left(A_{k}^{s} v_{1}, v_{2}\right)=a_{k}\left(v_{1}, A_{k}^{s} v_{2}\right) \quad \forall v_{1}, v_{2} \in V_{k}, s \in \mathbb{R} .
$$

2.1. $V$-cycle and $F$-cycle multigrid algorithms. The $k$-th level multigrid $V$ cycle and $F$-cycle algorithms are multilevel iterative methods for the equation

$$
A_{k} z=g \text {. }
$$

We assume that the finite element space $V_{k-1}$ is connected to $V_{k}$ by the (linear) intergrid transfer operator $I_{k-1}^{k}: V_{k-1} \longrightarrow V_{k}$, and denote its transpose with respect to the discrete inner products by $I_{k}^{k-1}: V_{k} \longrightarrow V_{k-1}$, i.e.,

$$
\left(I_{k}^{k-1} v, w\right)_{k-1}=\left(v, I_{k-1}^{k} w\right)_{k} \quad \forall v \in V_{k}, w \in V_{k-1} .
$$


The operator $P_{k}^{k-1}: V_{k} \longrightarrow V_{k-1}$ is the transpose of $I_{k-1}^{k}$ with respect to the nonconforming variational forms, i.e.,

$$
a_{k-1}\left(P_{k}^{k-1} v, w\right)=a_{k}\left(v, I_{k-1}^{k} w\right) \quad \forall v \in V_{k}, w \in V_{k-1} .
$$

These operators satisfy the well-known relation

$$
I_{k}^{k-1} A_{k}=A_{k-1} P_{k}^{k-1} .
$$

Finally we take $\Lambda_{k}$ to be a number dominating the spectral radius $\rho\left(A_{k}\right)$ of $A_{k}$.

Algorithm 2.1 (The $V$-cycle algorithm).

The $k$-th level symmetric $V$-cycle algorithm produces $M G_{\mathcal{V}}\left(k, g, z_{0}, m\right)$ as an approximate solution for (2.9) with initial guess $z_{0}$, where $m$ denotes the number of pre-smoothing and post-smoothing steps.

For $k=1$ we define

$$
M G_{\mathcal{V}}\left(1, g, z_{0}, m\right)=A_{1}^{-1} g .
$$

For $k \geq 2$ the approximate solution $M G_{\mathcal{V}}\left(k, g, z_{0}, m\right)$ is computed recursively in three steps:

Pre-smoothing. For $j=1, \ldots, m$, compute $z_{j}$ by

$$
z_{j}=z_{j-1}+\Lambda_{k}^{-1}\left(g-A_{k} z_{j-1}\right) .
$$

Coarse grid correction. Let $r_{k-1}=I_{k}^{k-1}\left(g-A_{k} z_{m}\right)$ and compute $z_{m+1}$ by

$$
z_{m+1}=z_{m}+I_{k-1}^{k} M G_{\mathcal{V}}\left(k-1, r_{k-1}, 0, m\right) .
$$

Post-smoothing. For $j=m+2, \ldots, 2 m+1$, compute $z_{j}$ by

$$
z_{j}=z_{j-1}+\Lambda_{k}^{-1}\left(g-A_{k} z_{j-1}\right) .
$$

We then define $M G_{\mathcal{V}}\left(k, g, z_{0}, m\right)=z_{2 m+1}$.

Algorithm 2.2. (The $F$-cycle algorithm)

The $k$-th level $F$-cycle algorithm (associated with the symmetric $V$-cycle algorithm) produces $M G_{\mathcal{F}}\left(k, g, z_{0}, m\right)$ as an approximate solution for (2.9).

For $k=1$, we define

$$
M G_{\mathcal{F}}\left(1, g, z_{0}, m\right)=A_{1}^{-1} g .
$$

For $k \geq 2$, we define $M G_{\mathcal{F}}\left(k, g, z_{0}, m\right)$ recursively in three steps:

Pre-smoothing. For $j=1, \ldots, m$, compute $z_{j}$ by

$$
z_{j}=z_{j-1}+\Lambda_{k}^{-1}\left(g-A_{k} z_{j-1}\right) .
$$

Coarse grid correction. Let $r_{k-1}=I_{k}^{k-1}\left(g-A_{k} z_{m}\right)$ and compute $z_{m+1}$ by

$$
\begin{aligned}
z_{m+\frac{1}{2}} & =M G_{\mathcal{F}}\left(k-1, r_{k-1}, 0, m\right), \\
z_{m+1} & =z_{m}+I_{k-1}^{k} M G_{\mathcal{V}}\left(k-1, r_{k-1}, z_{m+\frac{1}{2}}, m\right) .
\end{aligned}
$$

Post-smoothing. For $j=m+1, \ldots, 2 m+1$, compute $z_{j}$ by

$$
z_{j}=z_{j-1}+\Lambda_{k}^{-1}\left(g-A_{k} z_{j-1}\right) .
$$

We then define $M G_{\mathcal{F}}\left(k, g, z_{0}, m\right)=z_{2 m+1}$.

Remark 2.3. We use the Richardson relaxation scheme as the smoother for simplicity. The theory in this paper can be applied to other smoothers if the definition of the mesh-dependent norms in Section 3 is modified appropriately (cf. 23]). 
2.2. Error representations. Let $\mathbb{E}_{k, m}: V_{k} \longrightarrow V_{k}$ be the operator relating the initial error and the final error of the multigrid $V$-cycle algorithm applied to the equation (2.9), i.e.,

$$
\mathbb{E}_{k, m}\left(z-z_{0}\right)=z-M G_{\mathcal{V}}\left(k, g, z_{0}, m\right) .
$$

The operator $\mathbb{E}_{k, m}$ can be described in terms of the operators $I_{j-1}^{j}$ and $P_{j}^{j-1}$ $(2 \leq j \leq k)$ and the operators $R_{j}: V_{j} \longrightarrow V_{j}$ defined by

$$
R_{j}=I d_{j}-\Lambda_{j}^{-1} A_{j},
$$

where $I d_{j}: V_{j} \longrightarrow V_{j}$ is the identity operator. Clearly we have

$$
a_{j}\left(R_{j} v, w\right)=a_{j}\left(v, R_{j} w\right) \quad \forall v, w \in V_{j} .
$$

The following relations (cf. [36], [7]) are well known:

$$
\begin{aligned}
& \mathbb{E}_{k, m}=R_{k}^{m}\left[\left(I d_{k}-I_{k-1}^{k} P_{k}^{k-1}\right)+I_{k-1}^{k} \mathbb{E}_{k-1, m} P_{k}^{k-1}\right] R_{k}^{m} \quad \text { for } k \geq 2, \\
& \mathbb{E}_{1, m}=0 .
\end{aligned}
$$

Using (2.15) and (2.16), we obtain an additive expression for $\mathbb{E}_{k, m}$ :

$$
\begin{aligned}
\mathbb{E}_{k, m}= & R_{k}^{m}\left[\left(I d_{k}-I_{k-1}^{k} P_{k}^{k-1}\right)+I_{k-1}^{k} \mathbb{E}_{k-1, m} P_{k}^{k-1}\right] R_{k}^{m} \\
= & R_{k}^{m}\left(I d_{k}-I_{k-1}^{k} P_{k}^{k-1}\right) R_{k}^{m} \\
& \quad+R_{k}^{m} I_{k-1}^{k} R_{k-1}^{m}\left[\left(I d_{k-1}-I_{k-2}^{k-1} P_{k-1}^{k-2}\right)\right. \\
& \left.\quad+I_{k-2}^{k-1} \mathbb{E}_{k-2, m} P_{k-1}^{k-2}\right] R_{k-1}^{m} P_{k}^{k-1} R_{k}^{m} \\
= & \sum_{j=2}^{k} T_{k, j, m} R_{j}^{m}\left(I d_{j}-I_{j-1}^{j} P_{j}^{j-1}\right) R_{j}^{m} T_{j, k, m}
\end{aligned}
$$

where

$$
T_{k, k, m}=I d_{k},
$$

and for $j<k, T_{j, k, m}: V_{k} \longrightarrow V_{j}$ and $T_{k, j, m}: V_{j} \longrightarrow V_{k}$ are defined by

$$
\begin{aligned}
& T_{j, k, m}=P_{j+1}^{j} R_{j+1}^{m} \cdots P_{k}^{k-1} R_{k}^{m}, \\
& T_{k, j, m}=R_{k}^{m} I_{k-1}^{k} \cdots R_{j+1}^{m} I_{j}^{j+1} .
\end{aligned}
$$

Note that for $1 \leq j \leq k \leq \ell$ the following relations are valid:

$$
T_{j, \ell, m}=T_{j, k, m} T_{k, \ell, m} \quad \text { and } \quad T_{\ell, j, m}=T_{\ell, k, m} T_{k, j, m} .
$$

Also (2.11) and (2.14) imply

$$
a_{j}\left(T_{j, k, m} v, w\right)=a_{k}\left(v, T_{k, j, m} w\right) \quad \forall v \in V_{k}, w \in V_{j} .
$$

Let $\tilde{\mathbb{E}}_{k, m}: V_{k} \longrightarrow V_{k}$ be the operator relating the initial error and the final error of the $F$-cycle algorithm applied to the equation (2.9), i.e.,

$$
\tilde{\mathbb{E}}_{k, m}\left(z-z_{0}\right)=z-M G_{\mathcal{F}}\left(k, g, z_{0}, m\right) .
$$

The following relations (cf. 49]) are also well known:

$$
\begin{aligned}
\tilde{\mathbb{E}}_{1, m} & =0, \\
\tilde{\mathbb{E}}_{k, m} & =R_{k}^{m}\left[\left(I d_{k}-I_{k-1}^{k} P_{k}^{k-1}\right)+I_{k-1}^{k} \mathbb{E}_{k-1, m} \tilde{\mathbb{E}}_{k-1, m} P_{k}^{k-1}\right] R_{k}^{m}, k \geq 2 .
\end{aligned}
$$




\section{Assumptions}

In this section we state the assumptions for the convergence theory and derive some of their immediate consequences.

First we introduce a scale of mesh-dependent norms (cf. [4]) on each $V_{k}$ :

$$
\|v\|_{s, k}=\sqrt{\left(A_{k}^{s} v, v\right)_{k}} \quad \forall s \in \mathbb{R}, v \in V_{k} .
$$

It is clear from (2.4), (2.7) and (3.1) that

$$
\begin{aligned}
\|v\|_{0, k} & =\sqrt{(v, v)_{k}} & & \forall v \in V_{k}, \\
\|v\|_{1, k} & =\sqrt{a_{k}(v, v)}=\|v\|_{a_{k}} & & \forall v \in V_{k}, \\
\left\|A_{k}^{s} v\right\|_{t, k} & =\|v\|_{t+2 s, k} & & \forall v \in V_{k}, s, t \in \mathbb{R} .
\end{aligned}
$$

Moreover the Cauchy-Schwarz inequality implies

$$
\|v\|_{1+t, k}=\sup _{w \in V_{k} \backslash\{0\}} \frac{a_{k}(v, w)}{\|w\|_{1-t, k}} \quad \forall t \in \mathbb{R}, v \in V_{k} .
$$

To avoid the proliferation of constants we henceforth use the notation $A \lesssim B$ to represent the inequality $A \leq$ (constant) $\times B$, where the constant is independent of both the mesh (i.e., independent of the mesh size and the mesh level) and the number of smoothing steps. The statement $A \approx B$ is equivalent to $A \lesssim B$ and $B \lesssim A$.

Assumptions on $V_{k}$. We assume that

$$
\begin{array}{rlrl}
(v, v)_{k} & \approx\|v\|_{L_{2}(\Omega)}^{2} & & \forall v \in V_{k}, \\
\|v\|_{a_{k}} \lesssim h_{k}^{-1}\|v\|_{L_{2}(\Omega)} & & \forall v \in V_{k} .
\end{array}
$$

Assumptions on $I_{k-1}^{k}$ and $P_{k}^{k-1}$. We assume that $I_{k-1}^{k}$ and $P_{k}^{k-1}$ have the following properties:

$$
\begin{gathered}
\left\|I_{k-1}^{k} v\right\|_{1, k}^{2} \leq\left(1+\theta^{2}\right)\|v\|_{1, k-1}^{2}+C_{1} \theta^{-2} h_{k}^{2 \alpha}\|v\|_{1+\alpha, k-1}^{2} \\
\forall v \in V_{k-1}, \theta \in(0,1), \\
\left\|I_{k-1}^{k} v\right\|_{1-\alpha, k}^{2} \leq\left(1+\theta^{2}\right)\|v\|_{1-\alpha, k-1}^{2}+C_{2} \theta^{-2} h_{k}^{2 \alpha}\|v\|_{1, k-1}^{2} \\
\forall v \in V_{k-1}, \theta \in(0,1), \\
\left\|P_{k}^{k-1} v\right\|_{1-\alpha, k-1}^{2} \leq\left(1+\theta^{2}\right)\|v\|_{1-\alpha, k}^{2}+C_{3} \theta^{-2} h_{k}^{2 \alpha}\|v\|_{1, k}^{2} \\
\forall v \in V_{k}, \theta \in(0,1),
\end{gathered}
$$

where $\alpha$ is the index of elliptic regularity in (1.4) and the positive constants $C_{1}, C_{2}$ and $C_{3}$ are mesh-independent.

Remark 3.1. The estimates in 22 corresponding to (3.9) and (3.10) involve an index $\beta \in\left(0, \frac{1}{2}\right)$ instead of $\alpha$. Using the tools developed in Section 6 of this paper, one can also replace $\beta$ by $\alpha$ in [22]. 
Assumptions on $I_{k-1}^{k} P_{k}^{k-1}$ and $P_{k}^{k-1} I_{k-1}^{k}$. We assume the nonconforming finite element spaces have the following approximation properties:

$$
\begin{aligned}
\left\|\left(I d_{k}-I_{k-1}^{k} P_{k}^{k-1}\right) v\right\|_{1-\alpha, k} & \lesssim h_{k}^{2 \alpha}\|v\|_{1+\alpha, k} & & \forall v \in V_{k}, \\
\left\|\left(I d_{k-1}-P_{k}^{k-1} I_{k-1}^{k}\right) v\right\|_{1-\alpha, k-1} & \lesssim h_{k}^{\alpha}\|v\|_{1, k-1} & & \forall v \in V_{k-1} .
\end{aligned}
$$

We now derive some simple consequences of the assumptions above.

First we note that (3.6) and (3.7) imply

$$
\rho\left(A_{k}\right) \lesssim h_{k}^{-2}
$$

It follows easily (cf. [4, [36]) from (2.13), (3.1) and (3.13) that

$$
\begin{aligned}
\|v\|_{s, k} & \lesssim h_{k}^{t-s}\|v\|_{t, k} & & \forall v \in V_{k}, 0 \leq t \leq s \leq 2, \\
\left\|R_{k} v\right\|_{s, k} & \leq\|v\|_{s, k} & & \forall v \in V_{k}, s \in \mathbb{R}, \\
\left\|R_{k}^{m} v\right\|_{s, k} & \lesssim h_{k}^{t-s} m^{(t-s) / 2}\|v\|_{t, k} & & \forall v \in V_{k}, 0 \leq t \leq s \leq 2, m \geq 1 .
\end{aligned}
$$

The estimate (3.8) and (3.12) imply through (2.11), (3.5) and (3.14) three additional estimates:

$$
\begin{aligned}
\left\|I_{k-1}^{k} v\right\|_{1, k} & \lesssim\|v\|_{1, k-1} & & \forall v \in V_{k-1}, \\
\left\|P_{k}^{k-1} v\right\|_{1, k-1} & \lesssim\|v\|_{1, k} & & \forall v \in V_{k}, \\
\left\|\left(I d_{k-1}-P_{k}^{k-1} I_{k-1}^{k}\right) v\right\|_{1, k-1} & \lesssim h_{k}^{\alpha}\|v\|_{1+\alpha, k-1} & & \forall v \in V_{k-1} .
\end{aligned}
$$

Again, to avoid the proliferation of constants, we henceforth say that an estimate holds for $m$ sufficiently large if it is valid for $m \geq m_{*}$, where the positive integer $m_{*}$ is mesh-independent.

Lemma 3.2. Given any number $\omega \in(0,1)$, the following estimates hold for $m$ sufficiently large :

$$
\begin{aligned}
\left\|I_{k-1}^{k} R_{k-1}^{m} v\right\|_{1, k} & \leq(1+\omega)\|v\|_{1, k-1} & & \forall v \in V_{k-1}, \\
\left\|P_{k}^{k-1} R_{k}^{m} v\right\|_{1-\alpha, k-1} & \leq(1+\omega)\|v\|_{1-\alpha, k} & & \forall v \in V_{k}, \\
\left\|R_{k}^{m} I_{k-1}^{k} v\right\|_{1+\alpha, k} & \leq(1+\omega)\|v\|_{1+\alpha, k-1} & & \forall v \in V_{k-1} .
\end{aligned}
$$

Proof. From (2.1), (3.8), (3.15) and (3.16) we have

$$
\begin{aligned}
\left\|I_{k-1}^{k} R_{k-1}^{m} v\right\|_{1, k}^{2} & \leq\left(1+\omega^{2}\right)\left\|R_{k-1}^{m} v\right\|_{1, k-1}^{2}+C_{1} \omega^{-2} h_{k}^{2 \alpha}\left\|R_{k-1}^{m} v\right\|_{1+\alpha, k-1}^{2} \\
& \leq\left(1+\omega^{2}\right)\|v\|_{1, k-1}^{2}+C_{1}^{\prime} \omega^{-2} m^{-\alpha}\|v\|_{1, k-1}^{2},
\end{aligned}
$$

where the positive constant $C_{1}^{\prime}$ is independent of the mesh and the number of smoothing steps. The estimate (3.20) then follows if

$$
m^{\alpha} \geq \frac{C_{1}^{\prime} \omega^{-3}}{2} .
$$

Similarly we obtain (3.21) using (3.10), (3.15) and (3.16). The estimate (3.22) then follows from (2.11), (2.14), (3.5) and (3.21). 


\section{A strengthened Cauchy-Schwarz inequality}

In this section we derive a strengthened Cauchy-Schwarz inequality which takes into account the effect of smoothing. We begin by estimating the bounds of the operator $T_{j, k, m}$ with respect to various mesh-dependent norms. For brevity we will sometimes suppress the parameter $m$ and write $T_{j, k}$ instead of $T_{j, k, m}$.

From (2.18) $-(2.20)$, (3.21) and (3.22) we immediately have the following lemma.

Lemma 4.1. Let $j \leq k$. Given any $\omega \in(0,1)$, the following estimates hold for $m$ sufficiently large :

$$
\begin{array}{rlrl}
\left\|T_{j, k, m} v\right\|_{1-\alpha, j} & \leq(1+\omega)^{k-j}\|v\|_{1-\alpha, k} & & \forall v \in V_{k}, \\
\left\|T_{k, j, m} v\right\|_{1+\alpha, k} & \leq(1+\omega)^{k-j}\|v\|_{1+\alpha, j} & \forall v \in V_{j} .
\end{array}
$$

The next three lemmas and one corollary are preparatory for the crucial estimate involving $T_{K, k, m} T_{k, K, m}$ for $k \leq K$.

Lemma 4.2. Let $k \leq K$. Then the estimate

$$
\left\|T_{K, k, m} v\right\|_{1, K} \lesssim\|v\|_{1, k} \quad \forall v \in V_{k}
$$

holds for $m$ sufficiently large.

Proof. Let $v \in V_{k}$ be arbitrary. Given any $\omega \in(0,1)$, we have, from (1.8a), (2.11), (2.18), (2.20), (3.1), (3.3), (3.15), (3.19), (4.2) and the Cauchy-Schwarz inequality, that

$$
\begin{aligned}
\left\|T_{K, k} v\right\|_{1, K}^{2}= & a_{K}\left(T_{K, k} v, T_{K, k} v\right) \\
= & a_{K}\left(R_{K}^{m} I_{K-1}^{K} T_{K-1, k} v, R_{K}^{m} I_{K-1}^{K} T_{K-1, k} v\right) \\
\leq & a_{K}\left(I_{K-1}^{K} T_{K-1, k} v, I_{K-1}^{K} T_{K-1, k} v\right) \\
= & a_{K-1}\left(P_{K}^{K-1} I_{K-1}^{K} T_{K-1, k} v, T_{K-1, k} v\right) \\
= & a_{K-1}\left(T_{K-1, k} v, T_{K-1, k} v\right) \\
& \quad \quad \quad+a_{K-1}\left(\left[P_{K}^{K-1} I_{K-1}^{K}-I d_{K-1}\right] T_{K-1, k} v, T_{K-1, k} v\right) \\
\leq & \left\|T_{K-1, k} v\right\|_{1, K-1}^{2} \\
& \quad \quad \quad\left\|\left(P_{K}^{K-1} I_{K-1}^{K}-I d_{K-1}\right) T_{K-1, k} v\right\|_{1, K-1}\left\|T_{K-1, k} v\right\|_{1, K-1} \\
\leq & \left(1+\theta_{K}^{2}\right)\left\|T_{K-1, k} v\right\|_{1, K-1}^{2}+C_{\diamond} \theta_{K}^{-2} h_{K}^{2 \alpha}\left\|T_{K-1, k} v\right\|_{1+\alpha, K-1}^{2} \\
\leq & \left(1+\theta_{K}^{2}\right)\left\|T_{K-1, k} v\right\|_{1, K-1}^{2}+C_{\sharp} \theta_{K}^{-2}(1+\omega)^{2(K-k)} h_{K}^{2 \alpha}\|v\|_{1+\alpha, k}^{2}
\end{aligned}
$$

for all $v \in V_{k}$, provided $m$ is sufficiently large. Note that $\theta_{K} \in(0,1)$ is arbitrary, and the positive constant $C_{\sharp}$ is independent of the mesh, the number of smoothing steps and the parameters $\omega$ and $\theta_{K}$.

Iterating (4.4), we find

$$
\begin{aligned}
\left\|T_{K, k} v\right\|_{1, K}^{2} \leq & {\left[\prod_{k+1 \leq q \leq K}\left(1+\theta_{q}^{2}\right)\right]\|v\|_{1, k}^{2} } \\
+ & +C_{\sharp}\left[\sum_{k+1 \leq p \leq K}\left(\prod_{p+1 \leq q \leq K}\left(1+\theta_{q}^{2}\right)\right) \theta_{p}^{-2}(1+\omega)^{2(p-k)} h_{p}^{2 \alpha}\right]\|v\|_{1+\alpha, k}^{2},
\end{aligned}
$$

where $\theta_{k+1}, \ldots, \theta_{K}$ are arbitrary numbers in $(0,1)$. 
Note that $h_{p}=2^{k-p} h_{k}$ by (2.1). We now choose

$$
\omega=\left(\frac{4}{3}\right)^{\alpha / 2}-1
$$

so that

$$
(1+\omega)^{2(p-k)} h_{p}^{2 \alpha}=\left[(1+\omega)^{2} 4^{-\alpha}\right]^{p-k} h_{k}^{2 \alpha}=3^{-\alpha(p-k)} h_{k}^{2 \alpha},
$$

and then we take

$$
\theta_{q}=\left(\frac{2}{3}\right)^{\alpha(q-k) / 2} \quad \text { for } k+1 \leq q \leq K .
$$

Combining (4.5)-(4.7), we have

$$
\left\|T_{K, k} v\right\|_{1, K}^{2} \leq \rho_{1}\|v\|_{1, k}^{2}+C_{\sharp} \rho_{1} \rho_{2} h_{k}^{2 \alpha}\|v\|_{1+\alpha, k}^{2},
$$

where

$$
\rho_{1}=\prod_{j=1}^{\infty}\left[1+\left(\frac{2}{3}\right)^{\alpha \cdot j}\right]<\infty \quad \text { and } \quad \rho_{2}=\sum_{j=1}^{\infty}\left(\frac{1}{2}\right)^{\alpha \cdot j}<\infty .
$$

The estimate (4.3) follows from (3.14) and (4.8).

Lemma 4.2 (2.22) and (3.5) immediately imply the following corollary.

Corollary 4.3. Let $k \leq K$. Then the estimate

$$
\left\|T_{k, K, m} v\right\|_{1, k} \lesssim\|v\|_{1, K} \quad \forall v \in V_{K}
$$

holds for $m$ sufficiently large.

Lemma 4.4. Let $k \leq K$. Then the estimate

$$
\left\|T_{K, k, m} v\right\|_{1-\alpha, K} \lesssim\|v\|_{1-\alpha, k} \quad \forall v \in V_{k}
$$

holds for $m$ sufficiently large.

Proof. Let $v \in V_{k}$ be arbitrary. It follows from (2.18), (2.20), (3.9), (3.15) and (4.3) that, for $m$ sufficiently large,

$$
\begin{aligned}
\left\|T_{K, k} v\right\|_{1-\alpha, K}^{2} & =\left\|R_{K}^{m} I_{K-1}^{K} T_{K-1, k} v\right\|_{1-\alpha, K}^{2} \\
& \leq\left\|I_{K-1}^{K} T_{K-1, k} v\right\|_{1-\alpha, K}^{2} \\
& \leq\left(1+\theta_{K}^{2}\right)\left\|T_{K-1, k} v\right\|_{1-\alpha, K-1}^{2}+C_{2} \theta_{K}^{-2} h_{K}^{2 \alpha}\left\|T_{K-1, k} v\right\|_{1, K-1}^{2} \\
& \leq\left(1+\theta_{K}^{2}\right)\left\|T_{K-1, k} v\right\|_{1-\alpha, K-1}^{2}+C_{\dagger} \theta_{K}^{-2} h_{K}^{2 \alpha}\|v\|_{1, k}^{2}
\end{aligned}
$$

for any $\theta_{K} \in(0,1)$, where the positive constant $C_{\dagger}$ is independent of the mesh, the number of smoothing steps and the parameter $\theta_{K}$. Iterating (4.11), we find

$$
\begin{aligned}
\left\|T_{K, k} v\right\|_{1-\alpha, K}^{2} \leq & {\left[\prod_{k+1 \leq q \leq K}\left(1+\theta_{q}^{2}\right)\right]\|v\|_{1-\alpha, k}^{2} } \\
+ & +C_{\dagger}\left[\sum_{k+1 \leq p \leq K}\left(\prod_{p+1 \leq q \leq K}\left(1+\theta_{q}^{2}\right)\right) \theta_{p}^{-2} h_{p}^{2 \alpha}\right]\|v\|_{1, k}^{2},
\end{aligned}
$$

where $\theta_{k+1}, \ldots, \theta_{K}$ are arbitrary numbers in $(0,1)$.

As in the proof of Lemma 4.2 by choosing

$$
\theta_{q}=\left(\frac{1}{2}\right)^{\alpha(q-k) / 2} \quad \text { for } k+1 \leq q \leq K,
$$


we can deduce from (2.1) and (4.12) that

$$
\left\|T_{K, k} v\right\|_{1-\alpha, K}^{2} \lesssim\|v\|_{1-\alpha, k}^{2}+h_{k}^{2 \alpha}\|v\|_{1, k}^{2} .
$$

The estimate (4.10) now follows from (3.14) and (4.14).

Lemma 4.5. Let $k \leq K$. Then the estimate

$$
\left\|T_{k, K, m} v\right\|_{1-\alpha, k}^{2} \lesssim\|v\|_{1-\alpha, K}^{2}+h_{k}^{2 \alpha}\|v\|_{1, K}^{2} \quad \forall v \in V_{K}
$$

holds for $m$ sufficiently large.

Proof. Let $v \in V_{K}$ be arbitrary. From (2.19), (3.10), (3.15) and (4.9) we have, for $m$ sufficiently large,

$$
\begin{aligned}
& \left\|T_{k, K} v\right\|_{1-\alpha, k}^{2}=\left\|P_{k+1}^{k} R_{k+1}^{m} T_{k+1, K} v\right\|_{1-\alpha, k}^{2} \\
& \quad \leq\left(1+\theta_{k+1}^{2}\right)\left\|T_{k+1, K} v\right\|_{1-\alpha, k+1}^{2}+C_{3} \theta_{k+1}^{-2} h_{k+1}^{2 \alpha}\left\|T_{k+1, K} v\right\|_{1, k+1}^{2} \\
& \quad \leq\left(1+\theta_{k+1}^{2}\right)\left\|T_{k+1, K} v\right\|_{1-\alpha, k+1}^{2}+C_{b} \theta_{k+1}^{-2} h_{k+1}^{2 \alpha}\|v\|_{1, K}^{2},
\end{aligned}
$$

where $\theta_{k+1} \in(0,1)$ is arbitrary and the positive constant $C_{b}$ is independent of the mesh, the number of smoothing steps and the parameter $\theta_{k+1}$.

Iterating (4.16), we find

$$
\begin{aligned}
& \left\|T_{k, K} v\right\|_{1-\alpha, k}^{2} \leq\left[\prod_{k+1 \leq q \leq K}\left(1+\theta_{q}^{2}\right)\right]\|v\|_{1-\alpha, K}^{2} \\
& \quad+C_{b}\left[\sum_{k+1 \leq p \leq K}\left(\prod_{k+1 \leq q \leq p-1}\left(1+\theta_{q}^{2}\right)\right) \theta_{p}^{-2} h_{p}^{2 \alpha}\right]\|v\|_{1, K}^{2} .
\end{aligned}
$$

Choosing $\theta_{q}$ by formula (4.13), we deduce (4.15) from (2.1) and (4.17) as in the proof of Lemma 4.2 .

We can now establish a crucial estimate.

Lemma 4.6. Let $k \leq K$. Then the estimate

$$
\left\|T_{k, K, m} T_{K, k, m} v\right\|_{1-\alpha, k} \lesssim\|v\|_{1-\alpha, k} \quad \forall v \in V_{k}
$$

holds for $m$ sufficiently large.

Proof. It follows from (3.14), Lemma 4.2 Lemma 4.4 and Lemma 4.5 that

$$
\begin{gathered}
\left\|T_{k, K, m} T_{K, k, m} v\right\|_{1-\alpha, k}^{2} \lesssim\left\|T_{K, k, m} v\right\|_{1-\alpha, K}^{2}+h_{k}^{2 \alpha}\left\|T_{K, k, m} v\right\|_{1, K}^{2} \\
\lesssim\|v\|_{1-\alpha, k}^{2}+h_{k}^{2 \alpha}\|v\|_{1, k}^{2} \lesssim\|v\|_{1-\alpha, k}^{2},
\end{gathered}
$$

provided $m$ is sufficiently large.

The following proposition is the main result of this section.

Proposition 4.7 (Strengthened Cauchy-Schwarz inequality with smoothing). Let $1 \leq j \leq k \leq K$. Given any $\omega \in(0,1)$, the estimate

$$
\begin{aligned}
& a_{K}\left(T_{K, j, m} R_{j}^{q} v_{j}, T_{K, k, m} R_{k}^{q} v_{k}\right) \\
& \quad \lesssim q^{-\alpha}\left(\frac{1+\omega}{2^{\alpha}}\right)^{k-j}\left(h_{j}^{-\alpha}\left\|v_{j}\right\|_{1-\alpha, j}\right)\left(h_{k}^{-\alpha}\left\|v_{k}\right\|_{1-\alpha, k}\right)
\end{aligned}
$$

holds for all $v_{j} \in V_{j}$ and $v_{k} \in V_{k}$, provided $m$ is sufficiently large. 
Proof. Given any $\omega \in(0,1)$, from (2.1), (2.21), (2.22), (3.5), (3.15), (3.16), (4.1) and Lemma 4.6, we obtain

$$
\begin{aligned}
a_{K}\left(T_{K, j} R_{j}^{q}\right. & \left.v_{j}, T_{K, k} R_{k}^{q} v_{k}\right)=a_{j}\left(R_{j}^{q} v_{j}, T_{j, k} T_{k, K} T_{K, k} R_{k}^{q} v_{k}\right) \\
& \leq\left\|R_{j}^{q} v_{j}\right\|_{1+\alpha, j}\left\|T_{j, k} T_{k, K} T_{K, k} R_{k}^{q} v_{k}\right\|_{1-\alpha, j} \\
& \lesssim \frac{h_{j}^{-2 \alpha}}{q^{\alpha}}\left\|v_{j}\right\|_{1-\alpha, j}(1+\omega)^{k-j}\left\|v_{k}\right\|_{1-\alpha, k} \\
& =\frac{(1+\omega)^{k-j}}{q^{\alpha}}\left(\frac{h_{k}}{h_{j}}\right)^{\alpha}\left(h_{j}^{-\alpha}\left\|v_{j}\right\|_{1-\alpha, j}\right)\left(h_{k}^{-\alpha}\left\|v_{k}\right\|_{1-\alpha, k}\right) \\
& =q^{-\alpha}\left(\frac{1+\omega}{2^{\alpha}}\right)^{k-j}\left(h_{j}^{-\alpha}\left\|v_{j}\right\|_{1-\alpha, j}\right)\left(h_{k}^{-\alpha}\left\|v_{k}\right\|_{1-\alpha, k}\right),
\end{aligned}
$$

provided $m$ is sufficiently large.

Corollary 4.8. Let $v_{k} \in V_{k}$ for $1 \leq k \leq K$. Then the estimate

$$
a_{K}\left(\sum_{k=1}^{K} T_{K, k, m} R_{k}^{q} v_{k}, \sum_{k=1}^{K} T_{K, k, m} R_{k}^{q} v_{k}\right) \lesssim q^{-\alpha} \sum_{k=1}^{K} h_{k}^{-2 \alpha}\left\|v_{k}\right\|_{1-\alpha, k}^{2}
$$

holds for $m$ sufficiently large.

Proof. It follows from Proposition 4.7 that, given any $\omega \in(0,1)$,

$$
\begin{gathered}
a_{K}\left(\sum_{k=1}^{K} T_{K, k} R_{k}^{q} v_{k}, \sum_{k=1}^{K} T_{K, k} R_{k}^{q} v_{k}\right)=\sum_{j, k=1}^{K} a_{K}\left(T_{K, j} R_{j}^{q} v_{j}, T_{K, k} R_{k}^{q} v_{k}\right) \\
\lesssim q^{-\alpha} \sum_{j, k=1}^{K}\left(\frac{1+\omega}{2^{\alpha}}\right)^{|k-j|}\left(h_{j}^{-\alpha}\left\|v_{j}\right\|_{1-\alpha, j}\right)\left(h_{k}^{-\alpha}\left\|v_{k}\right\|_{1-\alpha, k}\right)
\end{gathered}
$$

for $m$ sufficiently large. We now choose $\omega$ so that $(1+\omega) 2^{-\alpha}<1$. The estimate (4.20) then follows from (4.21) and a discrete Young's inequality (cf. [37]).

\section{Convergence of $V$-CyCle And $F$-CyCle Algorithms}

In this section we establish the asymptotic behavior of the contraction numbers of $\mathbb{E}_{K, m}$ and $\tilde{\mathbb{E}}_{K, m}(K \geq 2)$ with respect to $m$.

The analysis in [22] for conforming $V$-cycle multigrid methods uses the fact that

$$
\left[I d_{k}-I_{k-1}^{k} P_{k}^{k-1}\right]^{2}=I d_{k}-I_{k-1}^{k} P_{k}^{k-1}
$$

in the case $V_{k-1} \subset V_{k} \subset H^{1}(\Omega)$. Since (5.1) does not hold for nonconforming finite element spaces, we need to consider first the contraction property of an auxiliary operator $\mathscr{E}_{K, m}: V_{K} \longrightarrow V_{K}$ defined by

$$
\begin{aligned}
\mathscr{E}_{K, m}=\sum_{k=2}^{K} T_{K, k, m}\left[R _ { k } ^ { m } \left(I d_{k}\right.\right. & \left.-I_{k-1}^{k} P_{k}^{k-1}\right) h_{k}^{-2 \alpha} A_{k}^{-\alpha} \\
& \left.\times\left(I d_{k}-I_{k-1}^{k} P_{k}^{k-1}\right) R_{k}^{m}\right] T_{k, K, m} .
\end{aligned}
$$

Lemma 5.1. The estimate

$$
\left\|\mathscr{E}_{K, m} v\right\|_{1, K} \lesssim m^{-\alpha}\|v\|_{1, K} \quad \forall v \in V_{K}
$$

holds for $m$ sufficiently large. 
Proof. Let $v \in V_{K}$ be arbitrary and

$$
v_{k}=\left(I d_{k}-I_{k-1}^{k} P_{k}^{k-1}\right) h_{k}^{-2 \alpha} A_{k}^{-\alpha}\left(I d_{k}-I_{k-1}^{k} P_{k}^{k-1}\right) R_{k}^{m} T_{k, K, m} v .
$$

We have, by (5.2), (5.4) and Corollary 4.8,

$$
\begin{aligned}
a_{K}\left(\mathscr{E}_{K, m} v, \mathscr{E}_{k, m} v\right) & =a_{K}\left(\sum_{k=2}^{K} T_{K, k, m} R_{k}^{m} v_{k}, \sum_{k=2}^{K} T_{K, k, m} R_{k}^{m} v_{k}\right) \\
& \lesssim m^{-\alpha} \sum_{k=2}^{K} h_{k}^{-2 \alpha}\left\|v_{k}\right\|_{1-\alpha, k}^{2}
\end{aligned}
$$

for $m$ sufficiently large. Moreover, (3.4), (3.11) and (5.4) imply

$$
\begin{aligned}
\left\|v_{k}\right\|_{1-\alpha, k} & \lesssim\left\|A_{k}^{-\alpha}\left(I d_{k}-I_{k-1}^{k} P_{k}^{k-1}\right) R_{k}^{m} T_{k, K, m} v\right\|_{1+\alpha, k} \\
& =\left\|A_{k}^{-\alpha / 2}\left(I d_{k}-I_{k-1}^{k} P_{k}^{k-1}\right) R_{k}^{m} T_{k, K, m} v\right\|_{1, k} .
\end{aligned}
$$

Let $w_{k}=\left(I d_{k}-I_{k-1}^{k} P_{k}^{k-1}\right) R_{k}^{m} T_{k, K, m} v$. It follows from (2.8), (2.11), (2.14), (2.22), (3.1) and (5.2) that

$$
\begin{aligned}
\sum_{k=2}^{K} h_{k}^{-2 \alpha} & \left\|A_{k}^{-\alpha / 2}\left(I d_{k}-I_{k-1}^{k} P_{k}^{k-1}\right) R_{k}^{m} T_{k, K, m} v\right\|_{1, k}^{2} \\
& =\sum_{k=2}^{K} h_{k}^{-2 \alpha} a_{k}\left(A_{k}^{-\alpha / 2} w_{k}, A_{k}^{-\alpha / 2}\left(I d_{k}-I_{k-1}^{k} P_{k}^{k-1}\right) R_{k}^{m} T_{k, K, m} v\right) \\
& =\sum_{k=2}^{K} a_{K}\left(T_{K, k, m} R_{k}^{m}\left(I d_{k}-I_{k-1}^{k} P_{k}^{k-1}\right) h_{k}^{-2 \alpha} A_{k}^{-\alpha} w_{k}, v\right) \\
& =a_{K}\left(\mathscr{E}_{K, m} v, v\right) .
\end{aligned}
$$

Combining (3.3), (5.5)-(5.7), and the Cauchy-Schwarz inequality, we find $\left\|\mathscr{E}_{K, m} v\right\|_{1, K}^{2}=a_{K}\left(\mathscr{E}_{K, m} v, \mathscr{E}_{K, m} v\right) \lesssim m^{-\alpha} a_{K}\left(\mathscr{E}_{K, m} v, v\right) \leq m^{-\alpha}\left\|\mathscr{E}_{k, m} v\right\|_{1, K}\|v\|_{1, K}$ and (5.3) follows.

We can now prove the convergence of the symmetric $V$-cycle algorithm.

Theorem 5.2 (Convergence of the symmetric $V$-cycle algorithm). There exist positive mesh-independent constants $C$ and $m_{*}$ such that

$$
\left\|\mathbb{E}_{K, m} v\right\|_{1, K} \leq \frac{C}{m^{\alpha}}\|v\|_{1, K} \quad \forall v \in V_{K}, K \geq 1, m \geq m_{*} .
$$

Proof. The case where $K=1$ is trivial. Let $v \in V_{K}(K \geq 2)$ be arbitrary and

$$
v_{k}=\left(I d_{k}-I_{k-1}^{k} P_{k}^{k-1}\right) R_{k}^{m} T_{k, K, m} v .
$$

Then (2.17), (5.9) and Corollary 4.8 imply that

$$
\begin{aligned}
a_{K}\left(\mathbb{E}_{K, m} v, \mathbb{E}_{K, m} v\right) & =a_{K}\left(\sum_{k=2}^{K} T_{K, k, m} R_{k}^{m} v_{k}, \sum_{k=2}^{K} T_{K, k, m} R_{k}^{m} v_{k}\right) \\
& \lesssim m^{-\alpha} \sum_{k=2}^{K} h_{k}^{-2 \alpha}\left\|v_{k}\right\|_{1-\alpha, k}^{2}
\end{aligned}
$$

for $m$ sufficiently large. 
From (3.4) and (5.9) we have

$$
\left\|v_{k}\right\|_{1-\alpha, k}=\left\|A_{k}^{-\alpha / 2}\left(I d_{k}-I_{k-1}^{k} P_{k}^{k-1}\right) R_{k}^{m} T_{k, K, m} v\right\|_{1, k} .
$$

Combining (3.3), (5.7), (5.10)-(5.11), Lemma 5.1 and the Cauchy-Schwarz inequality, we find

$$
\begin{aligned}
\left\|\mathbb{E}_{K, m} v\right\|_{1, K}^{2} & =a_{K}\left(\mathbb{E}_{K, m} v, \mathbb{E}_{K, m} v\right) \\
& \lesssim m^{-\alpha} a_{K}\left(\mathscr{E}_{K, m} v, v\right) \leq m^{-\alpha}\left\|\mathscr{E}_{k, m} v\right\|_{1, K}\|v\|_{1, K} \leq m^{-2 \alpha}\|v\|_{1, K}^{2}
\end{aligned}
$$

and (5.8) follows.

Finally we prove the convergence of the $F$-cycle algorithm.

Theorem 5.3 (Convergence of the $F$-cycle algorithm). There exist positive meshindependent constants $C$ and $m_{*}$ such that

$$
\left\|\tilde{\mathbb{E}}_{K, m} v\right\|_{1, K} \leq \frac{C}{m^{\alpha}}\|v\|_{1, K} \quad \forall v \in V_{K}, K \geq 1, m \geq m_{*} .
$$

Proof. Suppose that

$$
\left\|\tilde{\mathbb{E}}_{k-1, m} v\right\|_{1, k-1} \leq \eta_{k-1}\|v\|_{1, k-1} \quad \forall v \in V_{k-1} .
$$

From (2.24), (3.11), 3.15)-(3.18), (5.13) and Theorem 5.2 we have, for $m$ sufficiently large,

$$
\begin{aligned}
&\left\|\tilde{\mathbb{E}}_{k, m} v\right\|_{1, k} \leq\left\|R_{k}^{m}\left(I d_{k}-I_{k-1}^{k} P_{k}^{k-1}\right) R_{k}^{m} v\right\|_{1, k}+\left\|I_{k-1}^{k} \mathbb{E}_{k-1, m} \tilde{\mathbb{E}}_{k-1, m} P_{k}^{k-1} R_{k}^{m} v\right\|_{1, k} \\
& \quad \lesssim m^{-\alpha / 2} h_{k}^{-\alpha}\left\|\left(I d_{k}-I_{k-1}^{k} P_{k}^{k-1}\right) R_{k}^{m} v\right\|_{1-\alpha, k}+m^{-\alpha}\left\|\tilde{\mathbb{E}}_{k-1, m} P_{k}^{k-1} R_{k}^{m} v\right\|_{1, k-1} \\
& \quad \lesssim m^{-\alpha / 2} h_{k}^{\alpha}\left\|R_{k}^{m} v\right\|_{1+\alpha, k}+m^{-\alpha} \eta_{k-1}\left\|P_{k}^{k-1} R_{k}^{m} v\right\|_{1, k-1} \\
& \quad \lesssim m^{-\alpha}\left(1+\eta_{k-1}\right)\|v\|_{1, k} \forall v \in V_{k} .
\end{aligned}
$$

In other words we have,

$$
\left\|\tilde{\mathbb{E}}_{k, m} v\right\|_{1, k} \leq \eta_{k}\|v\|_{1, k} \quad \forall v \in V_{k}, m \geq m_{\dagger},
$$

where

$$
\eta_{k}=C_{\ddagger} m^{-\alpha}\left(1+\eta_{k-1}\right)
$$

and the positive constants $m_{\dagger}$ and $C_{\ddagger}$ are mesh-independent.

In view of (2.23) and (5.13)-(5.15), we can obtain by mathematical induction

$$
\left\|\tilde{\mathbb{E}}_{k, m} v\right\|_{1, k} \leq \frac{C_{\ddagger}}{m^{\alpha}-C_{\ddagger}}\|v\|_{1, k} \quad \forall v \in V_{k}, k \geq 1, m \geq m_{*},
$$

provided $m_{*} \geq \max \left(m_{\dagger}, C_{\ddagger}^{1 / \alpha}\right)$. The estimate (5.12) follows immediately from (5.16).

\section{AN ABSTRACT FRAMEWORK FOR NONCONFORMING MULTIGRID METHODS}

In order to apply the convergence results in Section 5 to a specific nonconforming multigrid method, one must verify the assumptions (3.6)-(3.12). This can be accomplished through the framework developed in [21. Indeed the standard discrete estimate (3.6) and inverse estimate (3.7) (cf. [30, 25]) are the assumptions (P) and (I) in [21], and (3.11) is established in Lemma 4.2 there.

The truly new ingredients among the assumptions in Section 3 are therefore the estimates (3.8) -(3.10) and (3.12). We will show in this section that they can be derived using the framework in [21] for second order problems and four additional 
conditions (cf. (6.10)-6.13) below). In the following discussion we will rely heavily on the results in 21$]$.

6.1. Results from 21] and new conditions. A key ingredient of the theory in 21] is the relation between the nonconforming finite element space $V_{k}$ and a conforming finite element space $\tilde{V}_{k} \subset H_{0}^{1}(\Omega)$ (referred to as a conforming relative of $V_{k}$ in [21]). These spaces are connected by the linear maps $E_{k}: V_{k} \longrightarrow \tilde{V}_{k}$ and $F_{k}: \tilde{V}_{k} \longrightarrow V_{k}$. Two of the properties of these maps are (cf. (FE) and Lemma 3.1 in 21]):

$$
\begin{aligned}
F_{k} \circ E_{k} & =I d_{k}, \\
\left\|F_{k} \tilde{v}\right\|_{L_{2}(\Omega)} & \lesssim\|\tilde{v}\|_{L_{2}(\Omega)} \quad \text { and } \quad\left\|F_{k} \tilde{v}\right\|_{a_{k}} \lesssim\|\tilde{v}\|_{H^{1}(\Omega)} \quad \forall \tilde{v} \in \tilde{V}_{k} .
\end{aligned}
$$

Let $\zeta \in H^{1+\alpha}(\Omega) \cap H_{0}^{1}(\Omega), \zeta_{k} \in V_{k}$ and $\zeta_{k-1} \in V_{k-1}$ be related by

$$
\begin{aligned}
a\left(\zeta, E_{k} v\right) & =a_{k}\left(\zeta_{k}, v\right) & & \forall v \in V_{k}, \\
a\left(\zeta, E_{k-1} v\right) & =a_{k-1}\left(\zeta_{k-1}, v\right) & & \forall v \in V_{k-1} .
\end{aligned}
$$

Then the following estimates are valid within the framework in [21] (cf. Theorem 3.5 and Lemma 3.7 there):

$$
\begin{aligned}
\left\|\zeta-\zeta_{k}\right\|_{a_{k}} & \lesssim h_{k}^{\alpha}\|\zeta\|_{H^{1+\alpha}(\Omega)}, \\
\left\|\Pi_{k} \zeta-\zeta_{k}\right\|_{1-\alpha, k} & \lesssim h_{k}^{2 \alpha}\|\zeta\|_{H^{1+\alpha}(\Omega)}, \\
\left\|\zeta_{k-1}-P_{k}^{k-1} \zeta_{k}\right\|_{1-\alpha, k-1} & \lesssim h_{k}^{2 \alpha}\|\zeta\|_{H^{1+\alpha}(\Omega)}
\end{aligned}
$$

where $\Pi_{k}: H_{0}^{1}(\Omega) \longrightarrow V_{k}$ is an interpolation operator.

The following estimates concerning $I_{k-1}^{k}$ and $\Pi_{k}$ are also established within the

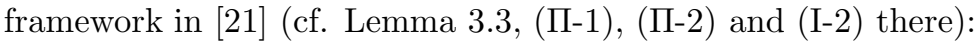

$$
\begin{aligned}
\left\|I_{k-1}^{k} v\right\|_{s, k} & \lesssim\|v\|_{s, k-1} & & \forall v \in V_{k-1}, 0 \leq s \leq 1, \\
\left\|\zeta-\Pi_{k} \zeta\right\|_{L_{2}(\Omega)} & \lesssim h_{k}|\zeta|_{H^{1}(\Omega)} & & \forall \zeta \in H_{0}^{1}(\Omega), \\
\left\|\zeta-\Pi_{k} \zeta\right\|_{a_{k}} & \lesssim h_{k}^{\alpha}\|\zeta\|_{H^{1+\alpha}(\Omega)} & & \forall \zeta \in H^{1+\alpha}(\Omega) \cap H_{0}^{1}(\Omega), \\
\left\|\Pi_{k} \zeta-I_{k-1}^{k} \Pi_{k-1} \zeta\right\|_{1-\alpha, k} & \lesssim h_{k}^{2 \alpha}\|\zeta\|_{H^{1+\alpha}(\Omega)} & & \forall \zeta \in H^{1+\alpha}(\Omega) \cap H_{0}^{1}(\Omega) .
\end{aligned}
$$

We will derive the estimates (3.8)- 3.10) and (3.12) using (6.1)-(6.9) and four additional conditions imposed on the intergrid transfer operator $I_{k-1}^{k}$ and the interpolation operator $\Pi_{k}$.

Four additional conditions. We assume that, in addition to the conditions (I-1) and (I-2) in [21,

$$
\left\|I_{k-1}^{k} v\right\|_{0, k}^{2} \leq\left(1+\theta^{2}\right)\|v\|_{0, k-1}^{2}+C_{0} \theta^{-2} h_{k}^{2 \alpha}\|v\|_{\alpha, k-1}^{2}
$$

for all $v \in V_{k-1}$ and $\theta \in(0,1)$, where the positive constant $C_{0}$ is mesh-independent.

Furthermore, the interpolation operator $\Pi_{k-1}: H_{0}^{1}(\Omega) \longrightarrow V_{k-1}$ actually maps

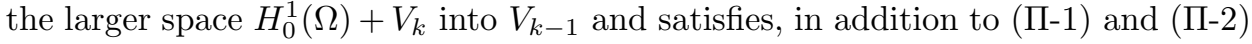
in [21], the estimates

$$
\begin{array}{ll}
\left\|\Pi_{k-1} v\right\|_{a_{k-1}} \lesssim\|v\|_{a_{k}} & \forall v \in H_{0}^{1}(\Omega)+V_{k}, \\
\left\|\Pi_{k-1} v-v\right\|_{L_{2}(\Omega)} \lesssim h_{k}\|v\|_{a_{k}} & \forall v \in V_{k}, \\
\left\|\Pi_{k-1} v\right\|_{0, k-1}^{2} \leq\left(1+\theta^{2}\right)\|v\|_{0, k}^{2}+C_{0}^{\prime} \theta^{-2} h_{k}^{2 \alpha}\|v\|_{\alpha, k}^{2} & \forall v \in V_{k}, \theta \in(0,1),
\end{array}
$$

where the positive constant $C_{0}^{\prime}$ is mesh-independent. 
6.2. Derivation of (3.8)-(3.10) and (3.12). We begin by introducing an operator $J_{k}$. Let $Q_{k}: L_{2}(\Omega) \longrightarrow \tilde{V}_{k}$ be the $L_{2}$-orthogonal projection operator. Then we define

$$
J_{k} \phi=F_{k} Q_{k} \phi \quad \forall \phi \in L_{2}(\Omega) .
$$

Lemma 6.1. The operator $J_{k}$ satisfies

$$
\begin{aligned}
J_{k} E_{k} v & =v & & \forall v \in V_{k}, \\
\left\|J_{k} \zeta\right\|_{1-\alpha, k} & \lesssim\|\zeta\|_{H^{1-\alpha}(\Omega)} & & \forall \zeta \in H_{0}^{1-\alpha}(\Omega) .
\end{aligned}
$$

Proof. The relation (6.15) follows immediately from (6.1) and (6.14).

From (3.2), (3.3), (3.6), (6.2) and standard properties of $Q_{k}$ (cf. [15]) we have

$$
\begin{array}{rlrl}
\left\|J_{k} \zeta\right\|_{0, k} \approx\left\|F_{k} Q_{k} \zeta\right\|_{L_{2}(\Omega)} \lesssim\left\|Q_{k} \zeta\right\|_{L_{2}(\Omega)} \lesssim\|\zeta\|_{L_{2}(\Omega)} & & \forall \zeta \in L_{2}(\Omega), \\
\left\|J_{k} \zeta\right\|_{1, k} & =\left\|F_{k} Q_{k} \zeta\right\|_{a_{k}} \lesssim\left\|Q_{k} \zeta\right\|_{H^{1}(\Omega)} \lesssim\|\zeta\|_{H^{1}(\Omega)} & & \forall \zeta \in H_{0}^{1}(\Omega) .
\end{array}
$$

The estimate (6.16) follows from (6.17), (6.18) and interpolation between Hilbert scales (cf. [48, [38], 16]).

Lemma 6.2. Given $\zeta_{k} \in V_{k}$, let $\zeta \in H_{0}^{1}(\Omega)$ be defined by

$$
a(\zeta, \phi)=a_{k}\left(\zeta_{k}, J_{k} \phi\right) \quad \forall \phi \in H_{0}^{1}(\Omega) .
$$

Then we have

$$
\begin{aligned}
a\left(\zeta, E_{k} v\right) & =a_{k}\left(\zeta_{k}, v\right) \quad \forall v \in V_{k}, \\
|\zeta|_{H^{1}(\Omega)} & \lesssim\left\|\zeta_{k}\right\|_{1, k}, \\
\|\zeta\|_{H^{1+\alpha}(\Omega)} & \lesssim\left\|\zeta_{k}\right\|_{1+\alpha, k}, \\
\left\|\Pi_{k} \zeta\right\|_{a_{k}} & \lesssim\left\|\zeta_{k}\right\|_{1, k} .
\end{aligned}
$$

Proof. The relation (6.20) follows immediately from (6.15) and (6.19).

From (3.3), (6.18), (6.19) and the Cauchy-Schwarz inequality we have

$$
a(\zeta, \zeta)=a_{k}\left(\zeta_{k}, J_{k} \zeta\right) \leq\left\|\zeta_{k}\right\|_{a_{k}}\left\|J_{k} \zeta\right\|_{a_{k}} \lesssim\left\|\zeta_{k}\right\|_{1, k}\|\zeta\|_{H^{1}(\Omega)} .
$$

which implies (6.21) in view of (2.6). The estimate (6.23) then follows immediately from (2.6), (6.11) and (6.21).

Using (3.5), (6.16) and (6.19), we find

$$
a_{k}\left(\zeta_{k}, J_{k} \phi\right) \leq\left\|\zeta_{k}\right\|_{1+\alpha, k}\left\|J_{k} \phi\right\|_{1-\alpha, k} \lesssim\left\|\zeta_{k}\right\|_{1+\alpha, k}\|\phi\|_{H^{1-\alpha}(\Omega)} \quad \forall \phi \in H_{0}^{1}(\Omega) .
$$

Thus the right-hand side of (6.19) defines a linear functional $F$ on $H_{0}^{1}(\Omega)$ which actually belongs to $H^{-1+\alpha}(\Omega)$ and

$$
\|F\|_{H^{-1+\alpha}(\Omega)} \lesssim\left\|\zeta_{k}\right\|_{1+\alpha, k} .
$$

The estimate (6.22) follows from (1.4) and (6.24).

We are now ready to derive the estimates (3.8)-(3.10) and (3.12). In the following derivations we use $C$ to denote a generic mesh-independent positive constant that is also independent of the parameter $\theta$.

Lemma 6.3. The estimate (3.8) holds. 
Proof. Let $\zeta_{k-1} \in V_{k-1}$ be arbitrary and $\zeta \in H_{0}^{1}(\Omega)$ be defined by

$$
a(\zeta, \phi)=a_{k-1}\left(\zeta_{k-1}, J_{k-1} \phi\right) \quad \forall \phi \in H_{0}^{1}(\Omega) .
$$

Then it follows from (1.8b), (2.1), (2.5), (3.3), (3.14), (6.3), (6.6), (6.8), (6.9), (6.25) and Lemma 6.2 that

$$
\begin{aligned}
\left\|I_{k-1}^{k} \zeta_{k-1}\right\|_{1, k}^{2} \leq & \left(\left\|\zeta_{k-1}\right\|_{a_{k}}+\left\|\zeta_{k-1}-I_{k-1}^{k} \zeta_{k-1}\right\|_{a_{k}}\right)^{2} \\
\leq & \left(1+\theta^{2}\right)\left\|\zeta_{k-1}\right\|_{a_{k-1}}^{2}+C \theta^{-2}\left(\left\|\zeta_{k-1}-\zeta\right\|_{a_{k}}^{2}+\left\|\zeta-\Pi_{k} \zeta\right\|_{a_{k}}^{2}\right. \\
& \left.\quad+\left\|\Pi_{k} \zeta-I_{k-1}^{k} \Pi_{k-1} \zeta\right\|_{a_{k}}^{2}+\left\|I_{k-1}^{k}\left(\Pi_{k-1} \zeta-\zeta_{k-1}\right)\right\|_{a_{k}}^{2}\right) \\
& \leq\left(1+\theta^{2}\right)\left\|\zeta_{k-1}\right\|_{1, k-1}^{2}+C \theta^{-2} h_{k}^{2 \alpha}\left\|\zeta_{k-1}\right\|_{1+\alpha, k-1}^{2} .
\end{aligned}
$$

Lemma 6.4. The estimate (3.9) holds.

Proof. Let $C_{*}$ be a constant that is greater than or equal to the constants $C_{0}$ and $C_{1}$ in (6.10) and (3.8), and define, for any $\theta \in(0,1)$,

$$
\left\langle v_{1}, v_{2}\right\rangle_{k-1, \theta}=\left(1+\theta^{2}\right)\left(v_{1}, v_{2}\right)_{k-1}+C_{*} \theta^{-2} h_{k}^{2 \alpha}\left(A_{k-1}^{\alpha} v_{1}, v_{2}\right)_{k-1}
$$

for all $v_{1}, v_{2} \in V_{k-1}$. Note that $A_{k-1}$ is symmetric positive definite with respect to the inner product $\langle\cdot, \cdot\rangle_{k-1, \theta}$.

It follows from (3.1), (3.8), (6.10) and (6.26) that

$$
\begin{array}{ll}
\left\|I_{k-1}^{k} v\right\|_{0, k}^{2} \leq\left\langle A_{k-1}^{0} v, v\right\rangle_{k-1, \theta} & \forall v \in V_{k-1}, \\
\left\|I_{k-1}^{k} v\right\|_{1, k}^{2} \leq\left\langle A_{k-1} v, v\right\rangle_{k-1, \theta} & \forall v \in V_{k-1},
\end{array}
$$

which imply through (3.1) and interpolation between Hilbert scales

$$
\left\|I_{k-1}^{k} v\right\|_{1-\alpha, k}^{2} \leq\left\langle A_{k-1}^{1-\alpha} v, v\right\rangle_{k-1, \theta}=\left(1+\theta^{2}\right)\|v\|_{1-\alpha, k-1}^{2}+C_{*} \theta^{-2} h_{k}^{2 \alpha}\|v\|_{1, k-1}^{2} .
$$

Lemma 6.5. It holds that

$$
\left\|\Pi_{k-1} v\right\|_{1, k-1}^{2} \leq\left(1+\theta^{2}\right)\|v\|_{1, k}^{2}+C_{\natural} \theta^{-2} h_{k}^{2 \alpha}\|v\|_{1+\alpha, k}^{2}
$$

for all $v \in V_{k}$ and $\theta \in(0,1)$, where the positive constant $C_{\natural}$ is mesh-independent.

Proof. Let $\zeta_{k} \in V_{k}$ be arbitrary and define $\zeta \in H_{0}^{1}(\Omega)$ by (6.19).

From (1.8b), (2.1), (2.5), (3.1), (6.3), (6.8), (6.11) and Lemma 6.2 we have

$$
\begin{aligned}
\left\|\Pi_{k-1} \zeta_{k}\right\|_{1, k-1}^{2} & \leq\left(\left\|\zeta_{k}\right\|_{a_{k}}+\left\|\zeta_{k}-\Pi_{k-1} \zeta_{k}\right\|_{a_{k}}\right)^{2} \\
& \leq\left(1+\theta^{2}\right)\left\|\zeta_{k}\right\|_{1, k}^{2}+C \theta^{-2}\left(\left\|\zeta_{k}-\zeta\right\|_{a_{k}}^{2}+\left\|\zeta-\Pi_{k-1} \zeta\right\|_{a_{k}}^{2}\right. \\
& \leq\left(1+\theta^{2}\right)\left\|\zeta_{k}\right\|_{1, k}^{2}+C \theta^{-2} h_{k}^{2 \alpha}\left\|\zeta_{k}\right\|_{1+\alpha, k}^{2} .
\end{aligned}
$$

The next lemma follows from (6.13), (6.27) and interpolation between Hilbert scales, as in the proof of Lemma 6.4

Lemma 6.6. It holds that

$$
\left\|\Pi_{k-1} v\right\|_{1-\alpha, k-1}^{2} \leq\left(1+\theta^{2}\right)\|v\|_{1-\alpha, k}^{2}+C_{\diamond} \theta^{-2} h_{k}^{2 \alpha}\|v\|_{1, k}^{2}
$$

for all $v \in V_{k}$ and $\theta \in(0,1)$, where the positive constant $C_{\diamond}$ is mesh-independent. 
We need one more estimate for the derivation of (B.10).

Lemma 6.7. The following estimate holds:

$$
\left\|\Pi_{k-1} \zeta-\Pi_{k-1} \Pi_{k} \zeta\right\|_{L_{2}(\Omega)} \lesssim h_{k}|\zeta|_{H^{1}(\Omega)} \quad \forall \zeta \in H_{0}^{1}(\Omega) .
$$

Proof. From (2.1), (2.6), (6.7), (6.11) and (6.12) we have

$$
\begin{aligned}
\left\|\Pi_{k-1} \zeta-\Pi_{k-1} \Pi_{k} \zeta\right\|_{L_{2}(\Omega)} & \leq\left\|\Pi_{k-1} \zeta-\zeta\right\|_{L_{2}(\Omega)}+\left\|\zeta-\Pi_{k} \zeta\right\|_{L_{2}(\Omega)} \\
& +\left\|\Pi_{k} \zeta-\Pi_{k-1} \Pi_{k} \zeta\right\|_{L_{2}(\Omega)} \\
& \lesssim h_{k}|\zeta|_{H^{1}(\Omega)}+h_{k}\left\|\Pi_{k} \zeta\right\|_{a_{k}} \lesssim h_{k}|\zeta|_{H^{1}(\Omega)} .
\end{aligned}
$$

Lemma 6.8. The estimate (3.10) holds.

Proof. Let $\zeta_{k} \in V_{k}$ be arbitrary. Define $\zeta \in H_{0}^{1}(\Omega)$ by (6.19) and $\zeta_{k-1} \in V_{k-1}$ by

$$
a_{k-1}\left(\zeta_{k-1}, v\right)=a\left(\zeta, E_{k-1} v\right) \quad \forall v \in V_{k-1} .
$$

We have, by (1.8b),

$$
\begin{aligned}
& \left\|P_{k}^{k-1} \zeta_{k}\right\|_{1-\alpha, k-1}^{2} \\
& \quad \leq\left(\left\|\Pi_{k-1} \Pi_{k} \zeta\right\|_{1-\alpha, k-1}+\left\|\Pi_{k-1} \Pi_{k} \zeta-P_{k}^{k-1} \zeta_{k}\right\|_{1-\alpha, k-1}\right)^{2} \\
& \quad \leq\left(1+\theta^{2}\right)\left\|\Pi_{k-1} \Pi_{k} \zeta\right\|_{1-\alpha, k-1}^{2}+C \theta^{-2}\left\|\Pi_{k-1} \Pi_{k} \zeta-P_{k}^{k-1} \zeta_{k}\right\|_{1-\alpha, k-1}^{2} .
\end{aligned}
$$

The first term on the right-hand side of (6.30) can be estimated using (1.8b), (3.3), (3.14), 6.4), (6.28) and Lemma 6.2

$$
\begin{aligned}
& \left\|\Pi_{k-1} \Pi_{k} \zeta\right\|_{1-\alpha, k-1}^{2} \leq\left(1+\theta^{2}\right)\left\|\Pi_{k} \zeta\right\|_{1-\alpha, k}^{2}+C \theta^{-2} h_{k}^{2 \alpha}\left\|\Pi_{k} \zeta\right\|_{1, k}^{2} \\
& \quad \leq\left(1+\theta^{2}\right)\left(\left\|\zeta_{k}\right\|_{1-\alpha, k}+\left\|\Pi_{k} \zeta-\zeta_{k}\right\|_{1-\alpha, k}\right)^{2}+C \theta^{-2} h_{k}^{2 \alpha}\left\|\zeta_{k}\right\|_{1, k}^{2} \\
& \quad \leq\left(1+\theta^{2}\right)^{2}\left\|\zeta_{k}\right\|_{1-\alpha, k}^{2}+C \theta^{-2} h_{k}^{4 \alpha}\|\zeta\|_{H^{1+\alpha}(\Omega)}^{2}+C \theta^{-2} h_{k}^{2 \alpha}\left\|\zeta_{k}\right\|_{1, k}^{2} \\
& \quad \leq\left(1+\theta^{2}\right)^{2}\left\|\zeta_{k}\right\|_{1-\alpha, k}^{2}+C \theta^{-2} h_{k}^{4 \alpha}\left\|\zeta_{k}\right\|_{1+\alpha, k}+C \theta^{-2} h_{k}^{2 \alpha}\left\|\zeta_{k}\right\|_{1, k}^{2} \\
& \quad \leq\left(1+\theta^{2}\right)^{2}\left\|\zeta_{k}\right\|_{1-\alpha, k}^{2}+C \theta^{-2} h_{k}^{2 \alpha}\left\|\zeta_{k}\right\|_{1, k}^{2} .
\end{aligned}
$$

Similarly the second term on the right-hand side of (6.30) can be estimated using (2.1), (3.2), (3.6), (3.14), (6.4), (6.5), (6.29) and Lemma 6.2:

$$
\begin{aligned}
& \left\|\Pi_{k-1} \Pi_{k} \zeta-P_{k}^{k-1} \zeta_{k}\right\|_{1-\alpha, k-1}^{2} \\
& \quad \leq\left(\left\|\Pi_{k-1}\left(\Pi_{k} \zeta-\zeta\right)\right\|_{1-\alpha, k-1}+\left\|\Pi_{k-1} \zeta-\zeta_{k-1}\right\|_{1-\alpha, k-1}\right. \\
& \left.\quad+\left\|\zeta_{k-1}-P_{k}^{k-1} \zeta_{k}\right\|_{1-\alpha, k-1}\right)^{2} \\
& \quad \leq C\left(h_{k}^{2(\alpha-1)}\left\|\Pi_{k-1}\left(\Pi_{k} \zeta-\zeta\right)\right\|_{0, k-1}^{2}+h_{k}^{2 \alpha}\left\|\zeta_{k}\right\|_{1, k}^{2}\right) \\
& \leq C h_{k}^{2 \alpha}\left\|\zeta_{k}\right\|_{1, k}^{2} .
\end{aligned}
$$

Combining (6.30)-(6.32), we have

$$
\left\|P_{k}^{k-1} \zeta_{k}\right\|_{1-\alpha, k-1}^{2} \leq\left(1+\theta^{2}\right)^{3}\left\|\zeta_{k}\right\|_{1-\alpha, k}^{2}+C \theta^{-2} h_{k}^{2 \alpha}\left\|\zeta_{k}\right\|_{1, k}^{2}
$$

which implies (3.10) since $\theta \in(0,1)$ is arbitrary.

We now turn to the derivation of (3.12) by first noting that the estimate

$$
\left\|P_{k}^{k-1} v\right\|_{1-\alpha, k-1} \lesssim\|v\|_{1-\alpha, k} \quad \forall v \in V_{k}
$$

follows from (3.10) and (3.14). 
Lemma 6.9. The estimate (3.12) holds.

Proof. Let $\zeta_{k-1} \in V_{k-1}$ be arbitrary and define $\zeta \in H_{0}^{1}(\Omega)$ and $\zeta_{k} \in V_{k}$ by 6.25 ) and (6.20), respectively. Then it follows from (2.1), (3.1), (3.5), (3.14), (6.4)-(6.6), (6.9), 6.33) and Lemma 6.2 that

$$
\begin{aligned}
a_{k-1}\left(\left(I d_{k-1}-P_{k}^{k-1} I_{k-1}^{k}\right) \zeta_{k-1}, w\right) \\
\quad=a_{k-1}\left(\zeta_{k-1}-P_{k}^{k-1} \zeta_{k}, w\right)-a_{k-1}\left(P_{k}^{k-1}\left(I_{k-1}^{k} \zeta_{k-1}-\zeta_{k}\right), w\right) \\
\quad \leq\left\|\zeta_{k-1}-P_{k}^{k-1} \zeta_{k}\right\|_{1-\alpha, k-1}\|w\|_{1+\alpha, k-1} \\
\quad+\left\|P_{k}^{k-1}\left(I_{k-1}^{k} \zeta_{k-1}-\zeta_{k}\right)\right\|_{1-\alpha, k-1}\|w\|_{1+\alpha, k-1} \\
\quad \lesssim h_{k}^{2 \alpha}\|\zeta\|_{H^{1+\alpha}(\Omega)}\|w\|_{1+\alpha, k-1}+\left(\left\|I_{k-1}^{k}\left(\zeta_{k-1}-\Pi_{k-1} \zeta\right)\right\|_{1-\alpha, k}\right. \\
\left.\quad \quad+\left\|I_{k-1}^{k} \Pi_{k-1} \zeta-\Pi_{k} \zeta\right\|_{1-\alpha, k}+\left\|\Pi_{k} \zeta-\zeta_{k}\right\|_{1-\alpha, k}\right)\|w\|_{1+\alpha, k-1} \\
\quad \lesssim h_{k}^{2 \alpha}\|\zeta\|_{H^{1+\alpha}(\Omega)}\|w\|_{1+\alpha, k-1} \\
\lesssim h_{k}^{2 \alpha}\left\|\zeta_{k-1}\right\|_{1+\alpha, k-1}\|w\|_{1+\alpha, k-1} \\
\lesssim h_{k}^{\alpha}\left\|\zeta_{k-1}\right\|_{1, k-1}\|w\|_{1+\alpha, k-1}
\end{aligned}
$$

for all $w \in V_{k-1}$, which implies (3.12) because of (3.5).

Remark 6.10. The proof of Lemma 6.9 actually establishes the stronger estimate

$$
\left\|\left(I d_{k-1}-P_{k}^{k-1} I_{k-1}^{k}\right) v\right\|_{1-\alpha, k-1} \lesssim h_{k}^{2 \alpha}\|v\|_{1+\alpha, k-1} \quad \forall v \in V_{k-1} .
$$

\section{Applications}

In this section we apply the theory developed in Sections 36 to two nonconforming finite element methods for the variational problem (1.1). As demonstrated in [21, both of these methods satisfy the assumptions of the framework developed in that paper. Therefore, from the discussion in Section 6 , it only remains for us to check the additional conditions (6.10) - 6.13) for these examples.

7.1. The nonconforming $\mathrm{P}_{1}$ finite element method. Let $e$ be (a segment of) an edge of $T \in \mathcal{T}_{k}$. We define

$$
M_{e, T}(v)=\left.\frac{1}{|e|} \int_{e} v\right|_{T} d s \quad \text { for } v \in H^{1}\left(\mathcal{T}_{\ell}\right), \ell \geq 1 .
$$

Remark 7.1. In the case where $\ell>k$ and $e$ is an edge of $T$, the integral on the right-hand side of (7.1) should be interpreted as

$$
\left.\int_{e} v\right|_{T} d s=\left.\sum_{j=1}^{2^{\ell-k}} \int_{e_{j}} v\right|_{T_{j}} d s
$$

where $e_{1}, \ldots, e_{2^{\ell-k}}$ are the edges from $\mathcal{T}_{\ell}$ that form a partition of $e$ and $T_{j} \subset T$ is a triangle in $\mathcal{T}_{\ell}$ with $e_{j}$ as an edge (cf. Figure 7.1 for the case $\ell-k=2$ ).

The nonconforming $\mathrm{P}_{1}$ finite element space $V_{k}$ is defined by (cf. 31])

$$
V_{k}=\left\{v \in L_{2}(\Omega):\left.v\right|_{T} \in\left\langle 1, x_{1}, x_{2}\right\rangle \forall T \in \mathcal{T}_{k}, M_{e, T}(v)=M_{e, T^{\prime}}(v) \text { if } e\right.
$$

is the common edge of $T$ and $T^{\prime}$ in $\mathcal{T}_{k}$, and $M_{e, T}(v)=0$

if $e \subset \partial \Omega$ is an edge of $T\}$. 


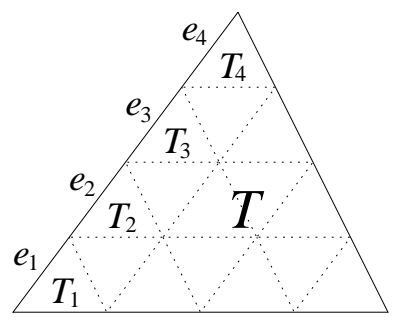

FiguRE 7.1. Interpretation of (7.1) when $\ell-k=2$

Henceforth we will denote $M_{e, T}(v)$ by $M_{e}(v)$ if there is no ambiguity about $T$ or if $v$ has an unambiguous mean value on $e$.

Let $\mathcal{E}_{k}$ be the set of the internal edges of $\mathcal{T}_{k}$. We define $(\cdot, \cdot)_{k}$ by

$$
\left(v_{1}, v_{2}\right)_{k}=h_{k}^{2} \sum_{e \in \mathcal{E}_{k}} M_{e}\left(v_{1}\right) M_{e}\left(v_{2}\right) \quad \forall v_{1}, v_{2} \in V_{k} .
$$

Let $\sum_{\ell=1}^{\infty} H^{1}\left(\mathcal{T}_{\ell}\right)$ be the subspace of $L_{2}(\Omega)$ whose members are finite sums of functions from the spaces $H^{1}\left(\mathcal{T}_{1}\right), H^{1}\left(\mathcal{T}_{2}\right), \ldots$ The weak interpolation operator $\Pi_{k}: \sum_{\ell=1}^{\infty} H^{1}\left(\mathcal{T}_{\ell}\right) \longrightarrow V_{k}$ is defined by

$$
M_{e}\left(\Pi_{k} v\right)=\frac{1}{2} \sum_{e \subset \partial T, T \in \mathcal{T}_{k}} M_{e, T}(v) \quad \forall e \in \mathcal{E}_{k} .
$$

Note that there are exactly two triangles in the sum and the mean value of $\Pi_{k} v$ on $e$ is just the average of the mean values of $v$ on $e$ from these two triangles.

The restriction of $\Pi_{k}$ to $H_{0}^{1}(\Omega)$ is precisely the weak interpolation operator used in [21] (cf. also [19, 6]), and the intergrid transfer operator $I_{k-1}^{k}: V_{k-1} \longrightarrow V_{k}$ in 21] is just the restriction of $\Pi_{k}$ to $V_{k-1}$.

The following lemma, a simple consequence of the Bramble-Hilbert lemma (cf. 8]) and scaling, is the key to the estimates (6.10)-(6.13).

Lemma 7.2. Let $e_{1}, e_{2}$ be two of the edges of $T \in \mathcal{T}_{k}$. Then we have

$$
\left|M_{e_{1}}(v)-M_{e_{2}}(v)\right| \lesssim|v|_{H^{1}(T)} \quad \forall v \in H^{1}(T) .
$$

Verification of (6.11). Let $e \in \mathcal{E}_{k-1}$ be the common edge of two triangles $T$ and $T^{\prime}$ in $\mathcal{T}_{k-1}$, and let $e_{1}$ (resp. $e_{2}$ ) be the half of the edge $e$ neighboring the two triangles $T_{1}$ and $T_{2}$ (resp. $T_{3}$ and $T_{4}$ ) in $\mathcal{T}_{k}$ (cf. Figure 7.2 ).

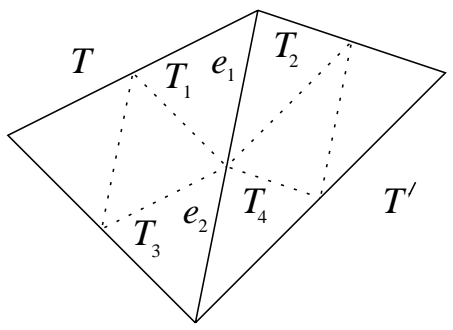

Figure 7.2. Two neighboring triangles in $\mathcal{T}_{k-1}$ 
Then for $v \in H_{0}^{1}(\Omega)+V_{k}$, we have

$$
M_{e, T}(v)=\frac{1}{2}\left[M_{e_{1}}(v)+M_{e_{2}}(v)\right]=M_{e, T^{\prime}}(v) .
$$

In view of (7.4) and (7.5), the interpolation operator $\Pi_{k-1}: H_{0}^{1}(\Omega)+V_{k} \longrightarrow V_{k-1}$ can be analyzed locally on each triangle of $\mathcal{T}_{k-1}$.

Let $T$ be a triangle in $\mathcal{T}_{k-1}$ subdivided into four triangles $T_{1}, \ldots, T_{4}$ and let $e_{1}$, $\ldots, e_{6}$ be the edges from $\mathcal{T}_{k}$ that are on $\partial T$ (cf. Figure 7.3). For any $v \in H_{0}^{1}(\Omega)+V_{k}$ we obtain from (7.4), (7.5) and scaling

$$
\left|\Pi_{k-1} v\right|_{H^{1}(T)}^{2} \lesssim \sum_{i, j=1}^{6}\left|M_{e_{i}}(v)-M_{e_{j}}(v)\right|^{2} .
$$

Note that Lemma 7.2 implies

$$
\left|M_{e_{i}}(v)-M_{e_{j}}(v)\right|^{2} \lesssim \sum_{\ell=1}^{4}|v|_{H^{1}\left(T_{\ell}\right)}^{2} \quad \forall v \in H^{1}(T)+V_{k}, 1 \leq i, j \leq 9 .
$$

For example, we have (cf. Figure 7.3)

$$
\begin{aligned}
\left|M_{e_{1}}(v)-M_{e_{4}}(v)\right|^{2} & \lesssim\left|M_{e_{1}}(v)-M_{e_{7}}(v)\right|^{2}+\left|M_{e_{7}}(v)-M_{e_{9}}(v)\right|^{2} \\
& +\left|M_{e_{9}}(v)-M_{e_{4}}(v)\right|^{2} \\
& \lesssim|v|_{H^{1}\left(T_{1}\right)}^{2}+|v|_{H^{1}\left(T_{4}\right)}^{2}+|v|_{H^{1}\left(T_{3}\right)}^{2} .
\end{aligned}
$$

It follows from (7.6) and (7.7) that

$$
\left|\Pi_{k-1} v\right|_{H^{1}(T)}^{2} \lesssim \sum_{\ell=1}^{4}|v|_{H^{1}\left(T_{\ell}\right)}^{2} \quad \forall v \in H^{1}(T)+V_{k} .
$$

We obtain by summing (7.8) over all the triangles in $\mathcal{T}_{k-1}$

$$
\sum_{T \in \mathcal{T}_{k-1}} \int_{T}\left|\nabla\left(\Pi_{k-1} v\right)\right|^{2} d x \lesssim \sum_{T \in \mathcal{T}_{k}} \int_{T}|\nabla v|^{2} d x \quad \forall v \in H_{0}^{1}(\Omega)+V_{k} .
$$

The estimate (6.11) then follows from (1.3), (2.3), (2.4), (7.9) and the Poincaré inequality for nonconforming $\mathrm{P}_{1}$ finite element functions (cf. 24] and the references therein).

Let $T$ be a generic triangle in $\mathcal{T}_{k-1}$ subdivided into four triangles $T_{1}, \ldots, T_{4} \in \mathcal{T}_{k}$ (cf. Figure 7.3). Henceforth we will denote by $e_{1}, \ldots, e_{9}$ the edges of $T_{1}, \ldots, T_{4}$ (cf.

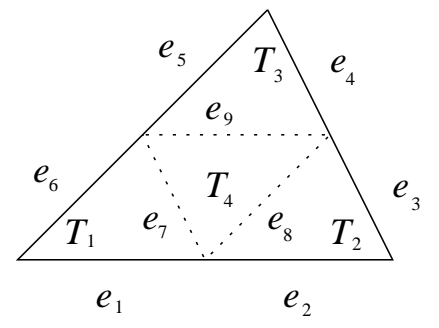

Figure 7.3. A divided triangle in $\mathcal{T}_{k-1}$ 
Figure 7.3$)$ and denote by $\tilde{e}_{j}(1 \leq j \leq 3)$ the edge of $T$ that contains the edges $e_{2 j-1}$ and $e_{2 j}$ from $\mathcal{T}_{k}$.

Verification of (6.12). Let $T$ be a generic triangle in $\mathcal{T}_{k-1}$. For any $v \in V_{k}$, we have by (7.4) and (7.5)

$$
M_{\tilde{e}_{j}}\left(\Pi_{k-1} v\right)=\frac{1}{2}\left[M_{e_{2 j-1}}(v)+M_{e_{2 j}}(v)\right] .
$$

Observe also that

$$
M_{\tilde{e}_{j}}(w)=\frac{1}{2}\left[M_{e_{2 j-1}}(w)+M_{e_{2 j}}(w)\right] \quad \forall w \in H^{1}(T),
$$

which together with (7.7) implies

$$
\left|M_{\tilde{e}_{j}}(w)-M_{e_{i}}(w)\right| \lesssim|w|_{H^{1}(T)} \quad \forall w \in H^{1}(T), 1 \leq j \leq 3,1 \leq i \leq 9 .
$$

We have by scaling

$$
\left\|\Pi_{k-1} v-v\right\|_{L_{2}(T)}^{2} \lesssim h_{k}^{2} \sum_{j=1}^{9}\left[M_{e_{j}}\left(\Pi_{k-1} v\right)-M_{e_{j}}(v)\right]^{2},
$$

and it follows from (7.7), (7.8), (7.10) and (7.11) that

$$
\begin{aligned}
\sum_{j=1}^{9}\left[M_{e_{j}}\left(\Pi_{k-1} v\right)-M_{e_{j}}(v)\right]^{2} & \lesssim \sum_{\ell=1}^{4}|v|_{H^{1}\left(T_{\ell}\right)}^{2}+\left|\Pi_{k-1} v\right|_{H^{1}(T)}^{2} \\
& \lesssim \sum_{\ell=1}^{4}|v|_{H^{1}\left(T_{\ell}\right)}^{2}
\end{aligned}
$$

Combining (7.12) and (7.13), we find

$$
\left\|\Pi_{k-1} v-v\right\|_{L_{2}(T)}^{2} \lesssim h_{k}^{2} \sum_{\ell=1}^{4}|v|_{H^{1}\left(T_{\ell}\right)}^{2} .
$$

In view of (1.3), (2.3) and (2.4), we obtain the estimate (6.12) by summing up the preceding estimate over all the triangles in $\mathcal{T}_{k-1}$.

Verification of ([6.13) . Denote by $\mathcal{E}_{T}$ the set of the three edges of $T$. Then we have by (7.2) and (7.3)

$$
(v, v)_{k}=\frac{h_{k}^{2}}{2} \sum_{T \in \mathcal{T}_{k}} \sum_{e \in \mathcal{E}_{T}}\left[M_{e}(v)\right]^{2} \quad \forall v \in V_{k} .
$$


The relation (7.14) allows us to focus on a generic $T \in \mathcal{T}_{k-1}$ in the derivation of (6.13). From (1.8), (7.7) and (7.10) we have

$$
\begin{aligned}
& \sum_{j=1}^{3}\left[M_{\tilde{e}_{j}}\left(\Pi_{k-1} v\right)\right]^{2} \leq \frac{1}{2} \sum_{i=1}^{6}\left[M_{e_{i}}(v)\right]^{2} \\
&=\frac{1}{4} \sum_{i=1}^{6}\left[M_{e_{i}}(v)\right]^{2}+\frac{1}{4} \sum_{j=1}^{3}\left\{\left(M_{e_{j+6}}(v)-\left[M_{e_{j+6}}(v)-M_{e_{2 j-1}}(v)\right]\right)^{2}\right. \\
&\left.+\left(M_{e_{j+6}}(v)-\left[M_{e_{j+6}}(v)-M_{e_{2 j}}(v)\right]\right)^{2}\right\} \\
&=\frac{1}{4} \sum_{i=1}^{6}\left[M_{e_{i}}(v)\right]^{2}+\frac{\left(1+\theta^{2}\right)}{2} \sum_{i=7}^{9}\left[M_{e_{i}}(v)\right]^{2}+C \theta^{-2} \sum_{\ell=1}^{4}|v|_{H^{1}\left(T_{\ell}\right)}^{2}
\end{aligned}
$$

for all $v \in V_{k}$ and $\theta \in(0,1)$, where the positive constant $C$ is mesh-independent.

Summing up the estimate (7.15) over all the triangles in $\mathcal{T}_{k-1}$ and using (1.3), (2.1), (2.3), (2.4), (3.2), (3.3), (7.2), (7.3) and (7.14), we arrive at

$$
\begin{aligned}
\left\|\Pi_{k-1} v\right\|_{0, k-1}^{2} & =\frac{h_{k-1}^{2}}{2} \sum_{T \in \mathcal{T}_{k-1}} \sum_{\tilde{e} \in \mathcal{E}_{T}}\left[M_{\tilde{e}}(v)\right]^{2} \\
& \leq\left(1+\theta^{2}\right) \frac{h_{k-1}^{2}}{4} \sum_{e \in \mathcal{E}_{k}}\left[M_{e}(v)\right]^{2}+C \theta^{-2} h_{k}^{2} \sum_{T \in \mathcal{T}_{k}}|v|_{H^{1}(T)}^{2} \\
& \leq\left(1+\theta^{2}\right)(v, v)_{k}+C \theta^{-2} h_{k}^{2}\|v\|_{a_{k}}^{2} \\
& =\left(1+\theta^{2}\right)\|v\|_{0, k}^{2}+C \theta^{-2} h_{k}^{2}\|v\|_{1, k}^{2} .
\end{aligned}
$$

The estimate (6.13) follows from (3.14) and (17.16).

Verification of (6.10). Let $e$ be an edge of $\mathcal{T}_{k}$ which is on the common boundary of $T, T^{\prime} \in \mathcal{T}_{k-1}$ (cf. Figure 7.2). Then we have by (1.8a) and (7.4)

$$
\left[M_{e}\left(I_{k-1}^{k} v\right)\right]^{2}=\frac{1}{4}\left[M_{e, T}(v)+M_{e, T^{\prime}}(v)\right]^{2} \leq \frac{1}{2}\left[M_{e, T}(v)\right]^{2}+\frac{1}{2}\left[M_{e, T^{\prime}}(v)\right]^{2} .
$$

For $T \in \mathcal{T}_{k-1}$, let $\mathcal{E}_{T}^{b}$ (resp. $\mathcal{E}_{T}^{i}$ ) denote the set of the edges from $\mathcal{T}_{k}$ that are on $\partial T$ (resp. interior to $T$ ). We obtain from (7.3) and (7.17) the estimate

$$
\left(I_{k-1}^{k} v, I_{k-1}^{k} v\right)_{k} \leq h_{k}^{2} \sum_{T \in \mathcal{T}_{k-1}}\left(\frac{1}{2} \sum_{e \in \mathcal{E}_{T}^{b}}\left[M_{e, T}(v)\right]^{2}+\sum_{e \in \mathcal{E}_{T}^{i}}\left[M_{e}(v)\right]^{2}\right)
$$

for all $v \in V_{k-1}$.

Again the estimate (7.18) allows us to focus on a generic $T \in \mathcal{T}_{k-1}$. It follows from (1.8b) and (7.11) that

$$
\frac{1}{2} \sum_{i=1}^{6}\left[M_{e_{i}}(v)\right]^{2}+\sum_{i=7}^{9}\left[M_{e_{i}}(v)\right]^{2} \leq 2\left(1+\theta^{2}\right) \sum_{j=1}^{3}\left[M_{\tilde{e}_{j}}(v)\right]^{2}+C \theta^{-2}|v|_{H^{1}(T)}^{2}
$$

for all $v \in H^{1}(T)$ and $\theta \in(0,1)$, where the positive constant $C$ is mesh-independent. 
Summing up the estimate (7.19) over all the triangles in $\mathcal{T}_{k-1}$, we find by (1.3), (2.1), (2.3), (2.4), (3.2), (3.3), (7.14) and (7.18)

$$
\begin{aligned}
\left\|I_{k-1}^{k} v\right\|_{0, k}^{2} & =\left(I_{k-1}^{k} v, I_{k-1}^{k} v\right)_{k} \\
& \leq h_{k}^{2} \sum_{T \in \mathcal{T}_{k-1}}\left(\frac{1}{2} \sum_{e \in \mathcal{E}_{T}^{b}}\left[M_{e, T}(v)\right]^{2}+\sum_{e \in \mathcal{E}_{T}^{i}}\left[M_{e}(v)\right]^{2}\right) \\
& \leq\left(1+\theta^{2}\right) \frac{h_{k-1}^{2}}{2} \sum_{T \in \mathcal{T}_{k-1}} \sum_{\tilde{e} \in \mathcal{E}_{T}}\left[M_{\tilde{e}}(v)\right]^{2}+C \theta^{-2} h_{k}^{2} \sum_{T \in \mathcal{T}_{k-1}}|v|_{H^{1}(T)}^{2} \\
& \leq\left(1+\theta^{2}\right)\|v\|_{0, k-1}^{2}+C \theta^{-2} h_{k}^{2}\|v\|_{1, k-1}^{2}
\end{aligned}
$$

for all $v \in V_{k-1}$ and $\theta \in(0,1)$.

The estimate (6.10) follows from (3.14) and (7.20).

7.2. The rotated $\mathrm{Q}_{1}$ finite element method. We shall adopt the notation in subsection 7.1 with obvious modifications. For simplicity we assume that $\mathcal{T}_{1}$ (and hence any $\mathcal{T}_{k}$ ) is a triangulation of $\Omega$ by rectangles whose sides are parallel to the coordinate axes. The rotated $\mathrm{Q}_{1}$ finite element space (cf. [42]) is defined by

$$
\begin{aligned}
V_{k}=\left\{v \in L_{2}(\Omega):\left.v\right|_{R} \in\left\langle 1, x_{1}, x_{2}, x_{1}^{2}-x_{2}^{2}\right\rangle \quad \forall R \in \mathcal{T}_{k}, M_{e, R}(v)=\right. \\
\\
M_{e, R^{\prime}}(v) \text { if } e \text { is the common edge of } R \text { and } R^{\prime} \text { in } \mathcal{T}_{k}, \text { and } M_{e, R}(v) \\
=0 \text { if } e \subset \partial \Omega \text { is an edge of } R\} .
\end{aligned}
$$

We define the inner product $(\cdot, \cdot)_{k}$ by

$$
\left(v_{1}, v_{2}\right)_{k}=h_{k}^{2} \sum_{e \in \mathcal{E}_{k}} M_{e}\left(v_{1}\right) M_{e}\left(v_{2}\right) \quad \forall v \in V_{k} .
$$

The weak interpolation operator $\Pi_{k}: \sum_{\ell=1}^{\infty} H^{1}\left(\mathcal{T}_{\ell}\right) \longrightarrow V_{k}$ is defined by

$$
M_{e}\left(\Pi_{k} v\right)=\frac{1}{2} \sum_{e \subset \partial R, R \in \mathcal{T}_{k}} M_{e}(v) \quad \forall e \in \mathcal{E}_{k} .
$$

The restriction of $\Pi_{k}$ to $H_{0}^{1}(\Omega)$ is precisely the weak interpolation operator used in [21], and the intergrid transfer operator $I_{k-1}^{k}: V_{k-1} \longrightarrow V_{k}$ used in [21] is just the restriction of $\Pi_{k}$ to $V_{k-1}$.

The following analog of Lemma 7.2 is again a simple consequence of the BrambleHilbert lemma and scaling.

Lemma 7.3. Let $e_{1}$ and $e_{2}$ be two edges of the rectangle $R \in \mathcal{T}_{k}$. Then we have

$$
\left|M_{e_{1}}(v)-M_{e_{2}}(v)\right| \lesssim|v|_{H^{1}(R)} \quad \forall v \in H^{1}(R) .
$$

Using Lemma 7.3 the verification of (6.10)- 6.13) proceeds as in subsection 7.1 and we therefore omit the details.

Remark 7.4. The results of this paper can also be applied to other nonconforming quadrilateral elements (cf. [33], 27], [28], 26]).

Remark 7.5. The nonconforming $\mathrm{P}_{1}$ and the rotated $\mathrm{Q}_{1}$ finite elements are equivalent to the lowest order triangular and rectangular Raviart-Thomas mixed finite elements (cf. 43, [2, 11). The results of this paper can therefore be applied to multigrid methods for the lowest order triangular and rectangular Raviart-Thomas mixed methods (cf. [20, [1]). 


\section{REFERENCES}

[1] T. Arbogast and Z. Chen, On the implementation of mixed methods as nonconforming methods for second order elliptic problems, Math. Comp. 64 (1995), 943-972. MR 95k:65102

[2] D.N. Arnold and F. Brezzi, Mixed and nonconforming finite element methods: implementation, postprocessing and error estimates, R.A.I.R.O Modél. Math. Anal. Numér. 19 (1985), 7-32. MR 87g:65126

[3] R.E. Bank and C.C. Douglas, Sharp estimates for multigrid rates of convergence with general smoothing and acceleration, SIAM J. Numer. Anal. 22 (1985), 617-633. MR 86j:65037

[4] R.E. Bank and T.F. Dupont, An optimal order process for solving finite element equations, Math. Comp. 36 (1981), 35-51. MR 82b:65113

[5] D. Braess and W. Hackbusch, A new convergence proof for the multigrid method including the $V$-cycle, SIAM J. Numer. Anal. 20 (1983), 967-975. MR 85h:65233

[6] D. Braess and R. Verfürth, Multigrid methods for nonconforming finite element methods, SIAM J. Numer. Anal. 27 (1990), 979-986. MR 91j:65164

[7] J.H. Bramble, Multigrid Methods, Longman Scientific \& Technical, Essex, 1993. MR 95b:65002

[8] J.H. Bramble and S.R. Hilbert, Estimation of linear functionals on Sobolev spaces with applications to Fourier transforms and spline interpolation, SIAM J. Numer. Anal. 7 (1970), 113-124. MR 41:7819

[9] J.H. Bramble and J.E. Pasciak, New convergence estimates for multigrid algorithms, Math. Comp. 49 (1987), 311-329. MR 89b:65234

[10] _ New estimates for multigrid algorithms including the V-cycle, Math. Comp. 60 (1993), 447-471. MR 94a:65064

[11] _ Uniform convergence estimates for multigrid $V$-cycle algorithms with less than full elliptic regularity, Domain Decomposition Methods in Science and Engineering (A. Quarteroni et al., ed.), Amer. Math. Soc., Providence, 1994, Contemporary Mathematics 157, pp. 17-26. MR 95f:65202

[12] J.H. Bramble, J.E. Pasciak, J. Wang, and J. Xu, Convergence estimates for multigrid algorithms without regularity assumptions, Math. Comp. 57 (1991), 23-45. MR 91m:65158

[13] _ Convergence estimates for product iterative methods with applications to domain decomposition and multigrid, Math. Comp. 57 (1991), 1-21. MR 92d:65094

[14] J.H. Bramble, J.E. Pasciak, and J. Xu, The analysis of multigrid algorithms with nonnested spaces or noninherited quadratic forms, Math. Comp. 56 (1991), 1-34. MR 91h:65159

[15] J.H. Bramble and J. Xu, Some estimates for a weighted $L^{2}$ projection, Math. Comp. 56 (1991), 463-476. MR 91k:65140

[16] J.H. Bramble and X. Zhang, The Analysis of Multigrid Methods, Handbook of Numerical Analysis, VII (P.G. Ciarlet and J.L. Lions, eds.), North-Holland, Amsterdam, 2000, pp. 173415. MR 2001m:65183

[17] A. Brandt, Multigrid solvers for non-elliptic and singular-perturbation steady-state problems, Weizmann Institute of Science, Rehovot, Israel, 1981.

[18] S.C. Brenner, Multigrid methods for nonconforming finite elements, Proceedings of the Fifth Copper Mountain Conference on Multigrid Methods (J. Mandel et al., ed.), SIAM, Philadelphia, 1989, pp. 54-65. MR 91h:65189

[19] _ An optimal-order multigrid method for P1 nonconforming finite elements, Math. Comp. 52 (1989), 1-15. MR 89f:65119

[20] _ A multigrid algorithm for the lowest-order Raviart-Thomas mixed triangular finite element method, SIAM J. Numer. Anal. 29 (1992), 647-678. MR 93j:65175

[21] - Convergence of nonconforming multigrid methods without full elliptic regularity, Math. Comp. 68 (1999), 25-53. MR 99c:65229

[22] - Convergence of the multigrid $V$-cycle algorithm for second order boundary value problems without full elliptic regularity, Math. Comp. 71 (2002), 507-525. MR 2003b:65132

[23] , Smoothers, mesh dependent norms, interpolation and multigrid, Appl. Numer. Math. 43 (2002), 45-56.

[24] Poincaré-Friedrichs inequalities for piecewise $H^{1}$ functions, SIAM J. Numer. Anal. 41 (2003), 306-324 (electronic).

[25] S.C. Brenner and L.R. Scott, The Mathematical Theory of Finite Element Methods (Second Edition), Springer-Verlag, New York-Berlin-Heidelberg, 2002. MR 2003a:65103 
[26] F. Brezzi, M. Fortin, and R. Stenberg, Error analysis of mixed-interpolated elements for Reissner-Mindlin plates, Math. Models and Methods in Appl. Sci. 1 (1991), 125-151. MR 92e:73030

[27] Z. Cai, J. Douglas, Jr., J.E. Santos, D. Sheen, and X. Ye, Nonconforming quadrilateral finite elements: a correction, Calcolo 37 (2000), 253-254. MR 2001k:65169

[28] Z. Cai, J. Douglas, Jr., and X. Ye, A stable nonconforming quadrilateral finite element method for the stationary Stokes and Navier-Stokes equations, Calcolo 36 (1999), 215-232. MR 2001b:65122

[29] Z. Chen, On the convergence of Galerkin-multigrid methods for nonconforming finite elements, East-West J. Numer. Math. 7 (1999), 79-104. MR 2000e:65116

[30] P.G. Ciarlet, The Finite Element Method for Elliptic Problems, North-Holland, Amsterdam, 1978. MR 58:25001

[31] M. Crouzeix and P.-A. Raviart, Conforming and nonconforming finite element methods for solving the stationary Stokes equations I, RAIRO Anal. Numér. 7 (1973), 33-75. MR 49:8401

[32] M. Dauge, Elliptic Boundary Value Problems on Corner Domains, Lecture Notes in Mathematics 1341, Springer-Verlag, Berlin-Heidelberg, 1988. MR 91a:35078

[33] J. Douglas, Jr., J.E. Santos, D. Sheen, and X. Ye, Nonconforming Galerkin methods based on quadrilateral elements for second order elliptic problems, $\mathrm{M}^{2} \mathrm{AN}$ Math. Model. Numer. Anal. 33 (1999), 747-770. MR 2000k:65206

[34] M. Griebel and P. Oswald, On the abstract theory of additive and multiplicative Schwarz algorithms, Numer. Math. 70 (1995), 163-180. MR 96a:65164

[35] W. Hackbusch, Multigrid convergence theory, Multigrid Methods (Lecture Notes in Mathematics 960) (W. Hackbusch and U. Trottenberg, eds.), Springer-Verlag, Berlin, 1982, pp. 177219. MR 84k:65113

[36] _ Multi-grid Methods and Applications, Springer-Verlag, Berlin-Heidelberg-New YorkTokyo, 1985. MR 87e:65082

[37] G.H. Hardy, J.E. Littlewood, and G. Pólya, Inequalities, Cambridge University Press, Cambridge, 1934.

[38] S.G. Kreĭn, Ju.I. Petunin, and E.M. Semenov, Interpolation of Linear Operators, Translations of Mathematical Monographs 54, American Mathematical Society, Providence, 1982. MR 84j:46103

[39] J. Mandel, S. McCormick, and R. Bank, Variational Multigrid Theory, Multigrid Methods, Frontiers In Applied Mathematics 3 (S. McCormick, ed.), SIAM, Philadelphia, 1987, pp. 131177.

[40] J. Mandel and S. Parter, On the multigrid F-cycle, Appl. Math. Comput. 37 (1990), 19-36. MR 91g:65266

[41] N. Neuss, V-cycle convergence with unsymmetric smoothers and applications to an anisotropic model problem, SIAM J. Numer. Anal. 35 (1998), 1201-1212. MR 99d:65109

[42] R. Rannacher and S. Turek, Simple nonconforming quadrilateral Stokes element, Numer. Meth. PDE 8 (1992), 97-111. MR 92i:65170

[43] P.-A. Raviart and J.M. Thomas, A mixed finite element method for second order elliptic problems, Mathematical Aspects of the Finite Element Method, Lecture Notes in Mathematics 606 (I.Galligani and E. Magenes, eds.), Springer-Verlag, Berlin, 1977, pp. 292-315. MR 58:3547

[44] Z. Shi and X. Xu, Cascadic multigrid method for elliptic problems, East-West J. Numer. Math 7 (1999), 199-209. MR 2000h:65185

[45] _ A V-cycle multigrid method for TRUNC plate element, Comput. Methods Appl. Mech. Engrg. 188 (2000), 483-493. MR 2001g:74059

[46] _ V-cycle multigrid methods for Wilson nonconforming element, Sci. China Ser. A 43 (2000), 673-684. MR 2002c:65217

[47] R. Stevenson, Nonconforming finite element and the cascadic multi-grid method, Numer. Math. 91 (2002), 351-387. MR 2003c:65139

[48] H. Triebel, Interpolation Theory, Function Spaces, Differential Operators, North-Holland, Amsterdam, 1978. MR 80i:46032a

[49] U. Trottenberg, C. Oosterlee, and A. Schüller, Multigrid, Academic Press, San Diego, 2001. MR 2002b:65002

[50] S. Turek, Efficient Solvers for Incompressible Flow Problems, Springer-Verlag, Berlin, 1999. MR 2000g:76071 
[51] P. Wesseling, An Introduction to Multigrid Methods, John Wiley \& Sons, Chichester, 1992. MR 93g:65006

[52] J. Xu, Convergence estimates for some multigrid algorithms, Third International Symposium on Domain Decomposition Methods for Partial Differential Equations, SIAM, Philadelphia, 1990, pp. 174-187. MR 91f:65194

[53] _ Iterative methods by space decomposition and subspace correction, SIAM Review 34 (1992), 581-613. MR 93k:65029

[54] X. Xu and L. Li, A note on convergence of V-cycle nonconforming multigrid methods, Appl. Math. Comput. 104 (1999), 191-206. MR 2000h:65186

[55] _ A V-cycle multigrid method for the plate bending problem discretized by nonconforming finite elements, J. Comput. Math. 17 (1999), 533-544. MR 2000k:65230

[56] H. Yserentant, Old and new convergence proofs for multigrid methods, Acta Numerica (1993), 285-326. MR 94i:65128

[57] X. Zhang, Multilevel Schwarz methods, Numer. Math. 63 (1992), 521-539. MR 93h:65047

[58] J. Zhao, Multigird Methods for Fourth Order Problems, Ph.D. thesis, University of South Carolina, in preparation.

Department of Mathematics, University of South Carolina, Columbia, South CarOLINA 29208

E-mail address: brenner@math.sc.edu 